Supporting Information

\title{
Correlating the Activity of Rhodium(I)-Phosphite-Lariat Ether Styrene Hydroformylation Catalysts with Alkali Metal Cation Binding Through NMR Spectroscopic Titration Methods
}

\author{
Justin R. Martin, Ethan C. Cagle, Aaron L. Lucius and Gary M. Gray* \\ Department of Chemistry, University of Alabama at Birmingham \\ $90114^{\text {th }}$ Street South, Birmingham, AL 35294-1240, USA \\ Email: gmgray@uab.edu
}

\section{Table of Contents}
(A) ${ }^{31} \mathrm{P}\left\{{ }^{1} \mathrm{H}\right\}$ and ${ }^{1} \mathrm{H}$ NMR Data for the Titrations of 2 and $3 \mathrm{With} \mathrm{LiBPh}_{4} \cdot 3 \mathrm{dme}$,

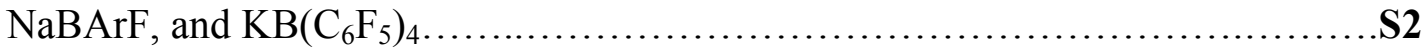
(B) FTIR Titrations of 2 and $\mathbf{3}$ With $\mathrm{LiBPh}_{4} \cdot 3 \mathrm{dme}, \mathrm{NaBArF}$, and $\left.\mathrm{KB}\left(\mathrm{C}_{6} \mathrm{~F}_{5}\right)_{4}\right) \ldots \ldots \ldots . . .513$
(C) NMR Spectra of New Ligands and Complexes.............................. 17

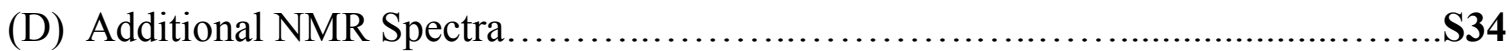


(A) ${ }^{31} \mathbf{P}\left\{{ }^{1} \mathrm{H}\right\}$ and ${ }^{1} \mathrm{H}$ NMR Data for the Titrations of 2 and 3 With $\mathrm{LiBPh}_{4} \cdot 3 \mathrm{dme}$, NaBArF, and $\operatorname{KB}\left(\mathrm{C}_{6} \mathrm{~F}_{5}\right)_{4}$.

Table S1. ${ }^{31} \mathrm{P}\left\{{ }^{1} \mathrm{H}\right\}$ NMR Data for the Titration of 2 with $\mathrm{LiBPh}_{4} \cdot 3 \mathrm{dme}$.

S1a: Data for upfield resonance.

[2] $=0.01 \mathrm{M} ;\left[\mathrm{Li}^{+}\right]=0.1 \mathrm{M} ;$ Initial ${ }^{31} \mathrm{P}\left\{{ }^{1} \mathrm{H}\right\}$ shift: $172.479 \mathrm{ppm}$

\begin{tabular}{|c|c|c|c|c|c|c|}
\hline $\begin{array}{l}\mathbf{V}_{\mathbf{L i}} \\
(\boldsymbol{\mu L})\end{array}$ & $\begin{array}{l}V_{\text {total }} \\
(\mu \mathbf{L})\end{array}$ & {$\left[\mathrm{Li}^{+}\right]_{\text {total }}$} & {$\left[\mathrm{Li}^{+}\right] /[\mathrm{MCE}]$} & $\begin{array}{l}{ }^{31} \mathbf{P}\left\{{ }^{1} H\right\} \\
\text { shift } \\
\text { (ppm) }\end{array}$ & $\begin{array}{l}\text { Normalized } \\
\text { signal }\end{array}$ & $\begin{array}{l}\text { Least } \\
\text { squares } \\
\text { fit }\end{array}$ \\
\hline 10 & 610 & 0.001639344 & 0.163934426 & 172.391 & 0.093298292 & 0.13951 \\
\hline 20 & 620 & 0.003225806 & 0.322580645 & 172.315 & 0.193166886 & 0.18377 \\
\hline 30 & 630 & 0.004761905 & 0.476190476 & 172.259 & 0.266754271 & 0.2426 \\
\hline 40 & 640 & 0.00625 & 0.625 & 172.195 & 0.350854139 & 0.30908 \\
\hline 50 & 650 & 0.007692308 & 0.769230769 & 172.151 & 0.408672799 & 0.3805 \\
\hline 60 & 660 & 0.009090909 & 0.909090909 & 172.114 & 0.457293035 & 0.45552 \\
\hline 65 & 665 & 0.009774436 & 0.977443609 & 172.099 & 0.477003942 & 0.49413 \\
\hline 70 & 670 & 0.010447761 & 1.044776119 & 172.073 & 0.511169514 & 0.53337 \\
\hline 75 & 675 & 0.011111111 & 1.111111111 & 172.04 & 0.554533509 & 0.57318 \\
\hline 80 & 680 & 0.011764706 & 1.176470588 & 172.003 & 0.603153745 & 0.61347 \\
\hline 90 & 690 & 0.013043478 & 1.304347826 & 171.931 & 0.697766097 & 0.69495 \\
\hline 100 & 700 & 0.014285714 & 1.428571429 & 171.875 & 0.771353482 & 0.77548 \\
\hline 110 & 710 & 0.015492958 & 1.549295775 & 171.821 & 0.842312746 & 0.84796 \\
\hline 120 & 720 & 0.016666667 & 1.666666667 & 171.789 & 0.884362681 & 0.89976 \\
\hline 130 & 730 & 0.017808219 & 1.780821918 & 171.767 & 0.913272011 & 0.92842 \\
\hline 140 & 740 & 0.018918919 & 1.891891892 & 171.75 & 0.935611038 & 0.94356 \\
\hline 150 & 750 & 0.02 & 2 & 171.743 & 0.944809461 & 0.95229 \\
\hline 160 & 760 & 0.021052632 & 2.105263158 & 171.732 & 0.959264126 & 0.95784 \\
\hline 170 & 770 & 0.022077922 & 2.207792208 & 171.728 & 0.964520368 & 0.96164 \\
\hline 180 & 780 & 0.023076923 & 2.307692308 & 171.713 & 0.984231275 & 0.96439 \\
\hline 200 & 800 & 0.025 & 2.5 & 171.719 & 0.976346912 & 0.96811 \\
\hline 260 & 860 & 0.030232558 & 3.023255814 & 171.716 & 0.980289093 & 0.97335 \\
\hline 400 & 1000 & 0.04 & 4 & 171.701 & 1 & 0.97716 \\
\hline
\end{tabular}


S1b: Data for downfield resonance.

$[2]=0.01 \mathrm{M} ;\left[\mathrm{Li}^{+}\right]=0.1 \mathrm{M} ;$ Initial ${ }^{31} \mathrm{P}\left\{{ }^{1} \mathrm{H}\right\}$ shift: $172.462 \mathrm{ppm}$

\begin{tabular}{|c|c|c|c|c|c|c|}
\hline $\begin{array}{l}\mathbf{V}_{\mathbf{L i}} \\
(\boldsymbol{\mu L})\end{array}$ & $\begin{array}{l}V_{\text {total }} \\
(\mu \mathrm{L})\end{array}$ & {$\left[\mathrm{Li}^{+}\right]_{\text {total }}$} & {$\left[\mathrm{Li}^{+}\right] /[\mathrm{MCE}]$} & $\begin{array}{l}{ }^{31} \mathbf{P}\left\{{ }^{1} \mathbf{H}\right\} \\
\text { shift } \\
(\mathrm{ppm})\end{array}$ & $\begin{array}{l}\text { Normalized } \\
\text { signal }\end{array}$ & $\begin{array}{l}\text { Least } \\
\text { squares } \\
\text { fit } \\
\end{array}$ \\
\hline 10 & 610 & 0.001639344 & 0.163934426 & 172.576 & -0.12467866 & -0.031784 \\
\hline 20 & 620 & 0.003225806 & 0.322580645 & 172.695 & -0.27763496 & -0.3751 \\
\hline 30 & 630 & 0.004761905 & 0.476190476 & 172.835 & -0.45758354 & -0.55008 \\
\hline 40 & 640 & 0.00625 & 0.625 & 172.95 & -0.60539845 & -0.61954 \\
\hline 50 & 650 & 0.007692308 & 0.769230769 & 173 & -0.66966581 & -0.60657 \\
\hline 60 & 660 & 0.009090909 & 0.909090909 & 172.95 & -0.60539845 & -0.52005 \\
\hline 65 & 665 & 0.009774436 & 0.977443609 & 172.894 & -0.53341902 & -0.44976 \\
\hline 70 & 670 & 0.010447761 & 1.044776119 & 172.786 & -0.39460154 & -0.36098 \\
\hline 75 & 675 & 0.011111111 & 1.111111111 & 172.647 & -0.21593830 & -0.25311 \\
\hline 80 & 680 & 0.011764706 & 1.176470588 & 172.49 & -0.01413881 & -0.1258 \\
\hline 90 & 690 & 0.013043478 & 1.304347826 & 172.294 & 0.237789203 & 0.1788 \\
\hline 100 & 700 & 0.014285714 & 1.428571429 & 172.116 & 0.466580977 & 0.47893 \\
\hline 110 & 710 & 0.015492958 & 1.549295775 & 171.982 & 0.638817481 & 0.66899 \\
\hline 120 & 720 & 0.016666667 & 1.666666667 & 171.909 & 0.732647815 & 0.76916 \\
\hline 130 & 730 & 0.017808219 & 1.780821918 & 171.848 & 0.811053985 & 0.82605 \\
\hline 140 & 740 & 0.018918919 & 1.891891892 & 171.822 & 0.844473008 & 0.86188 \\
\hline 150 & 750 & 0.02 & 2 & 171.792 & 0.883033419 & 0.88633 \\
\hline 160 & 760 & 0.021052632 & 2.105263158 & 171.773 & 0.907455013 & 0.90403 \\
\hline 170 & 770 & 0.022077922 & 2.207792208 & 171.758 & 0.926735219 & 0.91741 \\
\hline 180 & 780 & 0.023076923 & 2.307692308 & 171.748 & 0.939588689 & 0.92787 \\
\hline 200 & 800 & 0.025 & 2.5 & 171.736 & 0.955012853 & 0.94317 \\
\hline 260 & 860 & 0.030232558 & 3.023255814 & 171.716 & 0.980719794 & 0.96762 \\
\hline 400 & 1000 & 0.04 & 4 & 171.701 & 1 & 0.98799 \\
\hline
\end{tabular}


Table S2. Statistics for the ${ }^{31} \mathrm{P}\left\{{ }^{1} \mathrm{H}\right\}$ NMR Titration of 2 with $\mathrm{LiBPh}_{4} \cdot 3 \mathrm{dme}$.

S2a: Statistics for fit of upfield data.

Goodness-of-Fit

Statistics

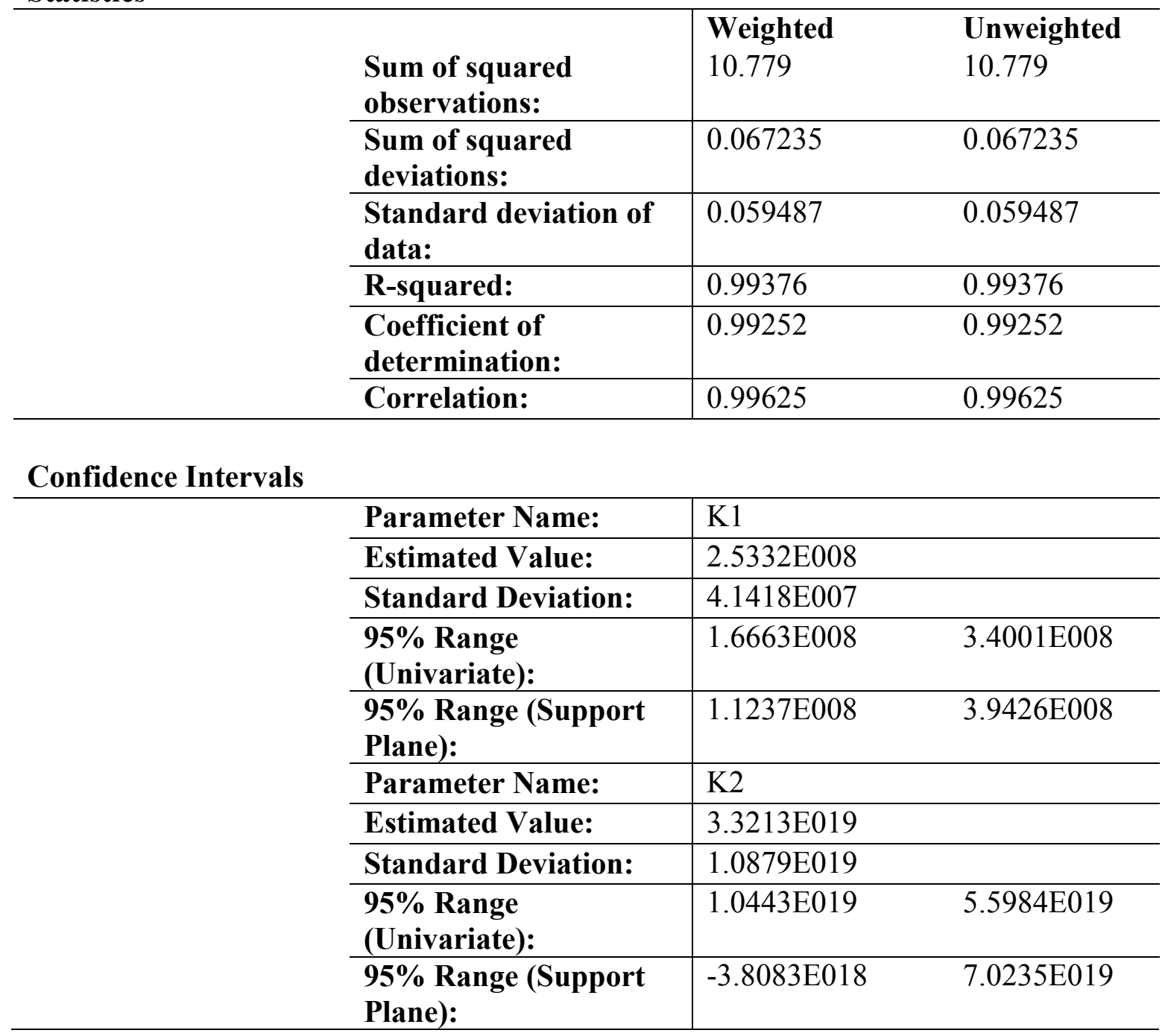


S2b: Statistics for fit of downfield data.

Goodness-of-Fit

Statistics

\begin{tabular}{l|lc}
\hline $\begin{array}{l}\text { Sum of squared } \\
\text { observations: }\end{array}$ & $\begin{array}{l}\text { Weighted } \\
12.686\end{array}$ & $\begin{array}{l}\text { Unweighted } \\
12.686\end{array}$ \\
\hline $\begin{array}{l}\text { Sum of squared } \\
\text { deviations: }\end{array}$ & 0.0065644 & 0.0065644 \\
\hline $\begin{array}{l}\text { Standard deviation of } \\
\text { data: }\end{array}$ & 0.018588 & 0.018588 \\
\hline R-squared: & 0.99948 & 0.99948 \\
\hline $\begin{array}{l}\text { Coefficient of } \\
\text { determination: }\end{array}$ & 0.99649 & 0.99649 \\
\hline Correlation: & 0.99825 & 0.99825 \\
\hline
\end{tabular}

\section{Confidence Intervals}

\begin{tabular}{l|lc} 
Parameter Name: & $\mathrm{K} 1$ & \\
\hline Estimated Value: & $2.5409 \mathrm{E} 008$ & $3.6066 \mathrm{E} 008$ \\
\hline Standard Deviation: & $5.0914 \mathrm{E} 007$ & $4.2735 \mathrm{E} 008$ \\
\hline $\begin{array}{l}\text { 95\% Range } \\
\text { (Univariate): }\end{array}$ & $1.4753 \mathrm{E} 008$ & \\
\hline $\begin{array}{l}\text { 95\% Range (Support } \\
\text { Plane): }\end{array}$ & $8.0833 \mathrm{E} 007$ & $2.2078 \mathrm{E} 19$ \\
\hline Parameter Name: & $\mathrm{K} 2$ & $2.8385 \mathrm{E} 019$ \\
\hline Estimated Value: & $1.2002 \mathrm{E} 19$ \\
\hline Standard Deviation: & $4.8124 \mathrm{E} 018$ \\
\hline $\begin{array}{l}\text { 95\% Range } \\
\text { (Univariate): }\end{array}$ & $1.9257 \mathrm{E} 018$ \\
\hline $\begin{array}{l}\text { 95\% Range (Support } \\
\text { Plane): }\end{array}$ & $-4.3808 \mathrm{E} 018$ \\
\hline
\end{tabular}


Table S3. ${ }^{31} \mathrm{P}\left\{{ }^{1} \mathrm{H}\right\}$ NMR Data for the Titration of $\mathbf{3}$ with $\mathrm{LiBPh}_{4} \cdot 3 \mathrm{dme}$.

$[3]=0.01 \mathrm{M} ;\left[\mathrm{Li}^{+}\right]=0.1 \mathrm{M}$; Initial ${ }^{31} \mathrm{P}\left\{{ }^{1} \mathrm{H}\right\}$ shift: $170.407 \mathrm{ppm}$

\begin{tabular}{|c|c|c|c|c|c|c|}
\hline $\begin{array}{l}V_{\mathbf{L i}} \\
(\mu \mathbf{L})\end{array}$ & $\begin{array}{l}V_{\text {total }} \\
(\mu \mathbf{L})\end{array}$ & {$\left[\mathrm{Li}^{+}\right]_{\text {total }}$} & {$\left[\mathrm{Li}^{+}\right] /[\mathrm{MCE}]$} & $\begin{array}{l}{ }^{31} \mathbf{P}\left\{{ }^{1} \mathbf{H}\right\} \\
\text { shift } \\
(\mathbf{p p m})\end{array}$ & $\begin{array}{l}\text { Normalized } \\
\text { signal }\end{array}$ & $\begin{array}{l}\text { Least } \\
\text { squares } \\
\text { fit }\end{array}$ \\
\hline 10 & 610 & 0.001639344 & 0.163934426 & 170.533 & -0.56756756 & 0.0012974 \\
\hline 20 & 620 & 0.003225806 & 0.322580645 & 170.692 & -1.28378378 & -1.5775 \\
\hline 30 & 630 & 0.004761905 & 0.476190476 & 170.88 & -2.13063063 & -2.5672 \\
\hline 40 & 640 & 0.00625 & 0.625 & 171.043 & -2.86486486 & -3.1902 \\
\hline 50 & 650 & 0.007692308 & 0.769230769 & 171.192 & -3.53603603 & -3.5307 \\
\hline 60 & 660 & 0.009090909 & 0.909090909 & 171.266 & -3.86936936 & -3.6252 \\
\hline 70 & 670 & 0.010447761 & 1.044776119 & 171.231 & -3.71171171 & -3.4847 \\
\hline 80 & 680 & 0.011764706 & 1.176470588 & 171.126 & -3.23873873 & -3.1012 \\
\hline 90 & 690 & 0.013043478 & 1.304347826 & 170.971 & -2.54054054 & -2.4574 \\
\hline 100 & 700 & 0.014285714 & 1.428571429 & 170.778 & -1.67117117 & -1.583 \\
\hline 110 & 710 & 0.015492958 & 1.549295775 & 170.579 & -0.77477477 & -0.72006 \\
\hline 120 & 720 & 0.016666667 & 1.6666666667 & 170.41 & -0.01351351 & -0.15288 \\
\hline 130 & 730 & 0.017808219 & 1.780821918 & 170.343 & 0.288288288 & 0.16775 \\
\hline 140 & 740 & 0.018918919 & 1.891891892 & 170.319 & 0.396396396 & 0.35944 \\
\hline 150 & 750 & 0.02 & 2 & 170.304 & 0.463963964 & 0.48417 \\
\hline 160 & 760 & 0.021052632 & 2.105263158 & 170.288 & 0.536036036 & 0.57113 \\
\hline 170 & 770 & 0.022077922 & 2.207792208 & 170.279 & 0.576576577 & 0.63501 \\
\hline 180 & 780 & 0.023076923 & 2.307692308 & 170.268 & 0.626126126 & 0.68383 \\
\hline 190 & 790 & 0.024050633 & 2.405063291 & 170.258 & 0.671171171 & 0.72233 \\
\hline 200 & 800 & 0.025 & 2.5 & 170.247 & 0.720720721 & 0.75346 \\
\hline 210 & 810 & 0.025925926 & 2.592592593 & 170.238 & 0.761261261 & 0.77913 \\
\hline 220 & 820 & 0.026829268 & 2.682926829 & 170.23 & 0.797297297 & 0.80066 \\
\hline 230 & 830 & 0.027710843 & 2.771084337 & 170.223 & 0.828828829 & 0.81898 \\
\hline 240 & 840 & 0.028571429 & 2.857142857 & 170.216 & 0.86036036 & 0.83475 \\
\hline 250 & 850 & 0.029411765 & 2.941176471 & 170.208 & 0.896396396 & 0.84847 \\
\hline 260 & 860 & 0.030232558 & 3.023255814 & 170.202 & 0.923423423 & 0.86052 \\
\hline 280 & 880 & 0.031818182 & 3.181818182 & 170.198 & 0.941441441 & 0.88068 \\
\hline 300 & 900 & 0.033333333 & 3.333333333 & 170.192 & 0.968468468 & 0.89687 \\
\hline 400 & 1000 & 0.04 & 4 & 170.185 & 1 & 0.94584 \\
\hline
\end{tabular}


Table S4. Statistics for the ${ }^{31} \mathrm{P}\left\{{ }^{1} \mathrm{H}\right\}$ NMR Titration of 3 with $\mathrm{LiBPh}_{4} \cdot 3 \mathrm{dme}$.

Goodness-of-Fit

Statistics

\begin{tabular}{l|ll}
$\begin{array}{l}\text { Sum of squared } \\
\text { observations: }\end{array}$ & $\begin{array}{l}\text { Weighted } \\
85.876\end{array}$ & $\begin{array}{l}\text { Unweighted } \\
85.876\end{array}$ \\
\hline $\begin{array}{l}\text { Sum of squared } \\
\text { deviations: }\end{array}$ & 0.92051 & 0.92051 \\
\hline $\begin{array}{l}\text { Standard deviation of } \\
\text { data: }\end{array}$ & 0.18464 & 0.18484 \\
\hline R-squared: & 0.98928 & 0.98928 \\
\hline $\begin{array}{l}\text { Coefficient of } \\
\text { determination: }\end{array}$ & 0.98837 & 0.98837 \\
\hline Correlation: & 0.99417 & 0.99417
\end{tabular}

\section{Confidence Intervals}

\begin{tabular}{l|lc} 
Parameter Name: & $\mathrm{K} 1$ & \\
\hline Estimated Value: & $7.945 \mathrm{E} 009$ & $8.1206 \mathrm{E} 009$ \\
\hline Standard Deviation: & $8.5735 \mathrm{E} 007$ & $8.1206 \mathrm{E} 009$ \\
\hline $\begin{array}{l}\text { 95\% Range } \\
\text { (Univariate): }\end{array}$ & $7.7694 \mathrm{E} 009$ & \\
\hline $\begin{array}{l}\text { 95\% Range (Support } \\
\text { Plane): }\end{array}$ & $7.7694 \mathrm{E} 009$ & \\
\hline Parameter Name: & $\mathrm{K} 2$ & $4.4651 \mathrm{E} 022$ \\
\hline Estimated Value: & $3.3995 \mathrm{E} 022$ & $4.7446 \mathrm{E} 022$ \\
\hline Standard Deviation: & $5.1936 \mathrm{E} 021$ & \\
\hline $\begin{array}{l}\text { 95\% Range } \\
\text { (Univariate): }\end{array}$ & $2.3338 \mathrm{E} 022$ \\
\hline $\begin{array}{l}\text { 95\% Range (Support } \\
\text { Plane): }\end{array}$ & $2.0543 \mathrm{E} 022$ & \\
\hline
\end{tabular}


Table S5. ${ }^{1} \mathrm{H}$ NMR Data for the Titration of 2 with $\mathrm{LiBPh}_{4} \cdot 3 \mathrm{dme}$.

$[2]=0.01 \mathrm{M} ;\left[\mathrm{Li}^{+}\right]=0.1 \mathrm{M}$; Initial ${ }^{1} \mathrm{H}$ shift: $3.880 \mathrm{ppm}$

\begin{tabular}{|c|c|c|c|c|c|c|}
\hline $\begin{array}{l}V_{\mathbf{L i}} \\
(\mu \mathbf{L})\end{array}$ & $\begin{array}{l}V_{\text {total }} \\
(\mu L)\end{array}$ & {$\left[\mathrm{Li}^{+}\right]_{\text {total }}$} & {$\left[\mathrm{Li}^{+}\right] /[\mathrm{MCE}]$} & $\begin{array}{l}{ }^{1} \text { H shift } \\
\text { (ppm) }\end{array}$ & $\begin{array}{l}\text { Normalized } \\
\text { signal }\end{array}$ & $\begin{array}{l}\text { Least } \\
\text { squares } \\
\text { fit }\end{array}$ \\
\hline 10 & 610 & 0.001639344 & 0.163934426 & 3.904 & -0.43636363 & 0.053661 \\
\hline 20 & 620 & 0.003225806 & 0.322580645 & 3.959 & -1.43636363 & -2.2069 \\
\hline 30 & 630 & 0.004761905 & 0.476190476 & 4.063 & -3.32727272 & -3.6317 \\
\hline 40 & 640 & 0.00625 & 0.625 & 4.144 & -4.8 & -4.527 \\
\hline 50 & 650 & 0.007692308 & 0.769230769 & 4.1728 & -5.32727272 & -5.0043 \\
\hline 60 & 660 & 0.009090909 & 0.909090909 & 4.1776 & -5.41818181 & -5.1046 \\
\hline 70 & 670 & 0.010447761 & 1.044776119 & 4.156 & -5.01818181 & -4.8239 \\
\hline 80 & 680 & 0.011764706 & 1.176470588 & 4.084 & -3.70909090 & -4.1146 \\
\hline 90 & 690 & 0.013043478 & 1.304347826 & 4.019 & -2.52727272 & -2.8996 \\
\hline 100 & 700 & 0.014285714 & 1.428571429 & 3.959 & -1.43636363 & -1.375 \\
\hline 110 & 710 & 0.015492958 & 1.549295775 & 3.911 & -0.56363636 & -0.37489 \\
\hline 120 & 720 & 0.016666667 & 1.6666666667 & 3.8809 & 0.072727273 & 0.087137 \\
\hline 130 & 730 & 0.017808219 & 1.780821918 & 3.8614 & 0.381818182 & 0.32659 \\
\hline 140 & 740 & 0.018918919 & 1.891891892 & 3.859 & 0.381818182 & 0.46993 \\
\hline 150 & 750 & 0.02 & 2 & 3.851 & 0.527272727 & 0.56478 \\
\hline 160 & 760 & 0.021052632 & 2.105263158 & 3.848 & 0.581818182 & 0.63204 \\
\hline 170 & 770 & 0.022077922 & 2.207792208 & 3.844 & 0.654545455 & 0.68216 \\
\hline 180 & 780 & 0.023076923 & 2.307692308 & 3.84 & 0.727272727 & 0.72094 \\
\hline 190 & 790 & 0.024050633 & 2.405063291 & 3.837 & 0.781818182 & 0.75183 \\
\hline 200 & 800 & 0.025 & 2.5 & 3.836 & 0.8 & 0.777 \\
\hline 300 & 900 & 0.033333333 & 3.333333333 & 3.829 & 0.927272727 & 0.89566 \\
\hline 400 & 1000 & 0.04 & 4 & 3.825 & 1 & 0.93725 \\
\hline
\end{tabular}


Table S6. Statistics for the ${ }^{1} \mathrm{H}$ NMR Titration of 2 with $\mathrm{LiBPh}_{4} \cdot 3 \mathrm{dme}$.

Goodness-of-Fit

Statistics

\begin{tabular}{l|ll}
$\begin{array}{l}\text { Sum of squared } \\
\text { observations: }\end{array}$ & $\begin{array}{l}\text { Weighted } \\
146.64\end{array}$ & $\begin{array}{l}\text { Unweighted } \\
146.64\end{array}$ \\
\hline $\begin{array}{l}\text { Sum of squared } \\
\text { deviations: }\end{array}$ & 2.5428 & 2.5428 \\
\hline $\begin{array}{l}\text { Standard deviation of } \\
\text { data: }\end{array}$ & 0.35657 & 0.35657 \\
\hline R-squared: & 0.98266 & 0.98266 \\
\hline $\begin{array}{l}\text { Coefficient of } \\
\text { determination: }\end{array}$ & 0.97746 & 0.97746 \\
\hline Correlation: & 0.98867 & 0.98867
\end{tabular}

\section{Confidence Intervals}

\begin{tabular}{l|lc} 
Parameter Name: & $\mathrm{K} 1$ & \\
\hline Estimated Value: & $2.0522 \mathrm{E} 008$ & \\
\hline Standard Deviation: & $6.2977 \mathrm{E} 006$ & $2.1836 \mathrm{E} 008$ \\
\hline $\begin{array}{l}\text { 95\% Range } \\
\text { (Univariate): }\end{array}$ & $1.9209 \mathrm{E} 008$ & $2.2187 \mathrm{E} 008$ \\
\hline $\begin{array}{l}\text { 95\% Range (Support } \\
\text { Plane): }\end{array}$ & $1.8858 \mathrm{E} 008$ & \\
\hline Parameter Name: & $\mathrm{K} 2$ & $5.651 \mathrm{E} 019$ \\
\hline Estimated Value: & $5.0091 \mathrm{E} 019$ \\
\hline Standard Deviation: & $3.0771 \mathrm{E} 018$ \\
\hline $\begin{array}{l}\text { 95\% Range } \\
\text { (Univariate): }\end{array}$ & $4.3672 \mathrm{E} 019$ \\
\hline $\begin{array}{l}\text { 95\% Range (Support } \\
\text { Plane): }\end{array}$ & $4.1958 \mathrm{E} 019$ & $5.8224 \mathrm{E} 019$ \\
\hline
\end{tabular}


Table S7. ${ }^{31} \mathrm{P}\left\{{ }^{1} \mathrm{H}\right\}$ NMR Data for the Titration of $\mathbf{3}$ with NaBArF.

$[3]=0.01 \mathrm{M} ;\left[\mathrm{Na}^{+}\right]=0.03 \mathrm{M}$; Initial ${ }^{31} \mathrm{P}\left\{{ }^{1} \mathrm{H}\right\}$ shift: $170.419 \mathrm{ppm}$

\begin{tabular}{|c|c|c|c|c|c|c|}
\hline $\begin{array}{l}\mathrm{V}_{\mathrm{Na}} \\
(\mu \mathrm{L})\end{array}$ & $\begin{array}{l}V_{\text {total }} \\
(\mu \mathrm{L})\end{array}$ & {$\left[\mathrm{Na}^{+}\right]_{\text {total }}$} & {$\left[\mathrm{Na}^{+}\right] /[\mathrm{MCE}]$} & $\begin{array}{l}{ }^{31} \mathbf{P}\left\{{ }^{1} \mathbf{H}\right\} \\
\text { shift } \\
(\mathbf{p p m})\end{array}$ & $\begin{array}{l}\text { Normalized } \\
\text { signal }\end{array}$ & $\begin{array}{l}\text { Least } \\
\text { squares } \\
\text { fit } \\
\end{array}$ \\
\hline 20 & 620 & 0.000967742 & 0.096774194 & 170.512 & 0.044797688 & 0.0060232 \\
\hline 40 & 640 & 0.001875 & 0.1875 & 170.685 & 0.128131021 & 0.13714 \\
\hline 60 & 660 & 0.002727273 & 0.272727273 & 170.8485 & 0.206888247 & 0.23948 \\
\hline 80 & 680 & 0.003529412 & 0.352941176 & 171.0205 & 0.289739884 & 0.3241 \\
\hline 100 & 700 & 0.004285714 & 0.428571429 & 171.184 & 0.36849711 & 0.39602 \\
\hline 120 & 720 & 0.005 & 0.5 & 171.3405 & 0.443882466 & 0.45815 \\
\hline 140 & 740 & 0.005675676 & 0.567567568 & 171.485 & 0.513487476 & 0.51241 \\
\hline 160 & 760 & 0.006315789 & 0.631578947 & 171.615 & 0.5761079 & 0.56017 \\
\hline 180 & 780 & 0.006923077 & 0.692307692 & 171.741 & 0.636801541 & 0.60245 \\
\hline 200 & 800 & 0.0075 & 0.75 & 171.831 & 0.680154143 & 0.64004 \\
\hline 220 & 820 & 0.00804878 & 0.804878049 & 171.897 & 0.71194605 & 0.6736 \\
\hline 240 & 840 & 0.008571429 & 0.857142857 & 171.942 & 0.733622351 & 0.70365 \\
\hline 260 & 860 & 0.009069767 & 0.906976744 & 171.968 & 0.746146435 & 0.73062 \\
\hline 280 & 880 & 0.009545455 & 0.954545455 & 171.98 & 0.751926782 & 0.75489 \\
\hline 300 & 900 & 0.01 & 1 & 172.002 & 0.762524085 & 0.77676 \\
\hline 320 & 920 & 0.010434783 & 1.043478261 & 172.041 & 0.781310212 & 0.79651 \\
\hline 340 & 940 & 0.010851064 & 1.085106383 & 172.0718 & 0.796146435 & 0.81437 \\
\hline 360 & 960 & 0.01125 & 1.125 & 172.0995 & 0.809489403 & 0.83055 \\
\hline 380 & 980 & 0.011632653 & 1.163265306 & 172.132 & 0.825144509 & 0.84522 \\
\hline 400 & 1000 & 0.012 & 1.2 & 172.168 & 0.842485549 & 0.85854 \\
\hline 460 & 1060 & 0.013018868 & 1.301886792 & 172.251 & 0.882466281 & 0.89172 \\
\hline 520 & 1120 & 0.013928571 & 1.392857143 & 172.309 & 0.910404624 & 0.91691 \\
\hline 600 & 1200 & 0.015 & 1.5 & 172.386 & 0.947495183 & 0.9416 \\
\hline 700 & 1300 & 0.016153846 & 1.615384615 & 172.459 & 0.98265896 & 0.96276 \\
\hline 800 & 1400 & 0.017142857 & 1.714285714 & 172.46325 & 0.984706166 & 0.97696 \\
\hline 900 & 1500 & 0.018 & 1.8 & 172.471 & 0.988439306 & 0.98671 \\
\hline 1000 & 1600 & 0.01875 & 1.875 & 172.4759 & 0.990799615 & 0.99355 \\
\hline 1100 & 1700 & 0.019411765 & 1.941176471 & 172.485 & 0.995183044 & 0.99845 \\
\hline 1200 & 1800 & 0.02 & 2 & 172.495 & 1 & 1.002 \\
\hline
\end{tabular}


Table S8. Statistics for the ${ }^{31} \mathrm{P}\left\{{ }^{1} \mathrm{H}\right\}$ NMR Titration of $\mathbf{3}$ with NaBArF.

Goodness-of-Fit

Statistics

\begin{tabular}{l|ll}
\hline $\begin{array}{l}\text { Sum of squared } \\
\text { observations: }\end{array}$ & $\begin{array}{l}\text { Weighted } \\
16.37\end{array}$ & $\begin{array}{l}\text { Unweighted } \\
16.37\end{array}$ \\
\hline $\begin{array}{l}\text { Sum of squared } \\
\text { deviations: }\end{array}$ & 0.012966 & 0.12966 \\
\hline $\begin{array}{l}\text { Standard deviation of } \\
\text { data: }\end{array}$ & 0.022773 & 0.022773 \\
\hline R-squared: & 0.99921 & 0.99921 \\
\hline $\begin{array}{l}\text { Coefficient of } \\
\text { determination: }\end{array}$ & 0.99387 & 0.99387 \\
\hline Correlation: & 0.99693 & 0.99693 \\
\hline
\end{tabular}

\section{Confidence Intervals}

\begin{tabular}{l|lc} 
Parameter Name: & $\mathrm{K} 1$ & \\
\hline Estimated Value: & $8.9154 \mathrm{E} 009$ & $1.5523 \mathrm{E} 010$ \\
\hline Standard Deviation: & $3.2083 \mathrm{E} 009$ & $1.9573 \mathrm{E} 010$ \\
\hline $\begin{array}{l}\text { 95\% Range } \\
\text { (Univariate): }\end{array}$ & $2.3078 \mathrm{E} 009$ & \\
\hline $\begin{array}{l}\text { 95\% Range (Support } \\
\text { Plane): }\end{array}$ & $-1.7421 \mathrm{E} 009$ & \\
\hline Parameter Name: & $\mathrm{K} 2$ & $1.1171 \mathrm{E} 021$ \\
\hline Estimated Value: & $4.5002 \mathrm{E} 20$ & $1.5259 \mathrm{E} 021$ \\
\hline Standard Deviation: & $3.2388 \mathrm{E} 020$ & \\
\hline $\begin{array}{l}\text { 95\% Range } \\
\text { (Univariate): }\end{array}$ & $-2.1702 \mathrm{E} 020$ \\
\hline $\begin{array}{l}\text { 95\% Range (Support } \\
\text { Plane): }\end{array}$ & $-6.2586 \mathrm{E} 020$ & \\
\hline
\end{tabular}


Table S9. ${ }^{31} \mathrm{P}\left\{{ }^{1} \mathrm{H}\right\}$ NMR Data for the Titration of 3 with $\mathrm{KB}\left(\mathrm{C}_{6} \mathrm{~F}_{5}\right)_{4}$.

$[3]=0.01 \mathrm{M} ;\left[\mathrm{K}^{+}\right]=0.03 \mathrm{M}$; Initial ${ }^{31} \mathrm{P}\left\{{ }^{1} \mathrm{H}\right\}$ shift: $170.419 \mathrm{ppm}$

\begin{tabular}{|c|c|c|c|c|c|c|c|}
\hline $\begin{array}{l}\mathbf{V}_{\mathbf{K}} \\
\mu \mathbf{L}\end{array}$ & $\begin{array}{l}V_{\text {total }} \\
\mu \mathrm{L}\end{array}$ & {$\left[\mathbf{K}^{+}\right]_{\text {total }}$} & $\begin{array}{l}{\left[\mathbf{K}^{+}\right] /} \\
{[\mathbf{M C E}]}\end{array}$ & $\begin{array}{l}\text { downfield } \\
{ }^{31} \mathbf{P}\left\{{ }^{1} \mathbf{H}\right\} \\
\text { shift } \\
\text { (ppm) }\end{array}$ & $\begin{array}{l}\text { Normalized } \\
\text { signal }\end{array}$ & $\begin{array}{l}\text { upfield } \\
{ }^{31} \mathbf{P}\left\{{ }^{1} \mathbf{H}\right\} \\
\text { shift } \\
\text { (ppm) }\end{array}$ & $\begin{array}{l}\text { Normalized } \\
\text { signal }\end{array}$ \\
\hline 20 & 620 & 0.000967 & 0.09677 & 3.855 & 0.11351351 & - & - \\
\hline 40 & 640 & 0.001875 & 0.1875 & 3.876 & 0.22702702 & - & - \\
\hline 60 & 660 & 0.002727 & 0.27272 & 3.894 & 0.32432432 & - & - \\
\hline 80 & 680 & 0.003529 & 0.35294 & 3.91 & 0.41081081 & - & - \\
\hline 100 & 700 & 0.004285 & 0.42857 & 3.929 & 0.51351351 & - & - \\
\hline 120 & 720 & 0.005 & 0.5 & 3.943 & 0.58918918 & - & - \\
\hline 140 & 740 & 0.005675 & 0.56756 & 3.951 & 0.63243243 & 4.018 & 0.53958944 \\
\hline 160 & 760 & 0.006315 & 0.63157 & 3.96 & 0.68108108 & 4.041 & 0.60703812 \\
\hline 180 & 780 & 0.006923 & 0.69230 & 3.969 & 0.72972973 & 4.067 & 0.68328445 \\
\hline 200 & 800 & 0.0075 & 0.75 & 3.977 & 0.77297297 & 4.088 & 0.74486803 \\
\hline 220 & 820 & 0.008048 & 0.80487 & 3.994 & 0.86486486 & 4.099 & 0.7771261 \\
\hline 240 & 840 & 0.008571 & 0.85714 & 4 & 0.89729729 & 4.119 & 0.83577712 \\
\hline 260 & 860 & 0.009069 & 0.90697 & 4.006 & 0.92972973 & 4.142 & 0.90322580 \\
\hline 280 & 880 & 0.009545 & 0.95454 & 4.015 & 0.97837837 & 4.168 & 0.97947214 \\
\hline 300 & 900 & 0.01 & 1 & 4.021 & 1.01081081 & 4.18 & 1.01466275 \\
\hline 320 & 920 & 0.010434 & 1.04347 & 4.02 & 1.00540540 & 4.185 & 1.02932551 \\
\hline 340 & 940 & 0.010851 & 1.08510 & 4.018 & 0.99459459 & 4.182 & 1.02052785 \\
\hline 400 & 1000 & 0.012 & 1.2 & 4.017 & 0.98918918 & 4.179 & 1.01173020 \\
\hline 500 & 1100 & 0.013636 & 1.36363 & 4.019 & 1 & 4.175 & 1 \\
\hline
\end{tabular}


(B) FTIR Titrations of $1-3$ With $\mathrm{LiBPh}_{4} \cdot 3 \mathrm{dme}$, NaBArF, and $\mathrm{KB}\left(\mathrm{C}_{6} \mathrm{~F}_{5}\right)_{4}$

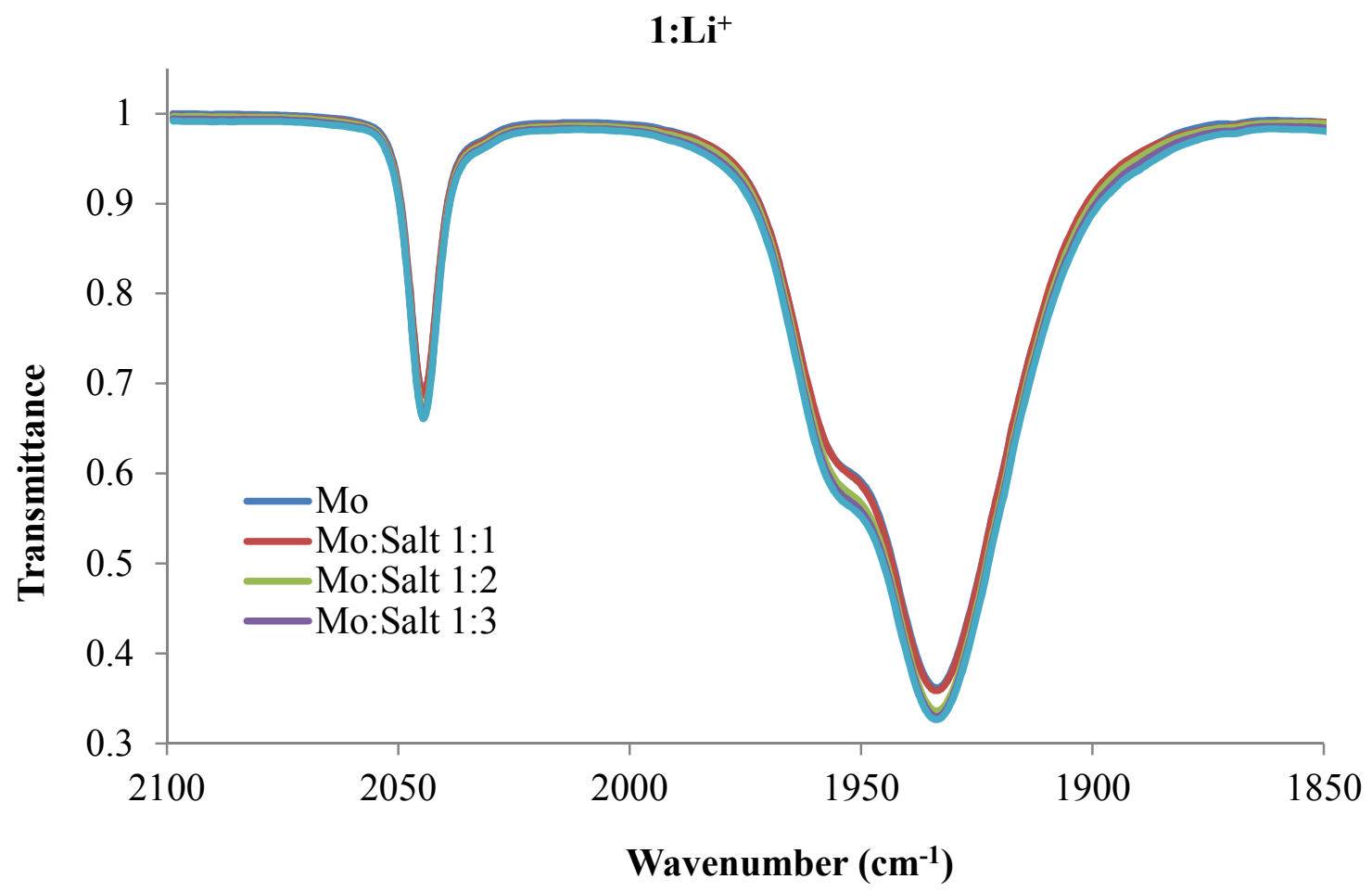

Figure S1. FTIR titration of 1 with $\mathrm{LiBPh}_{4} \cdot 3 \mathrm{dme}$.

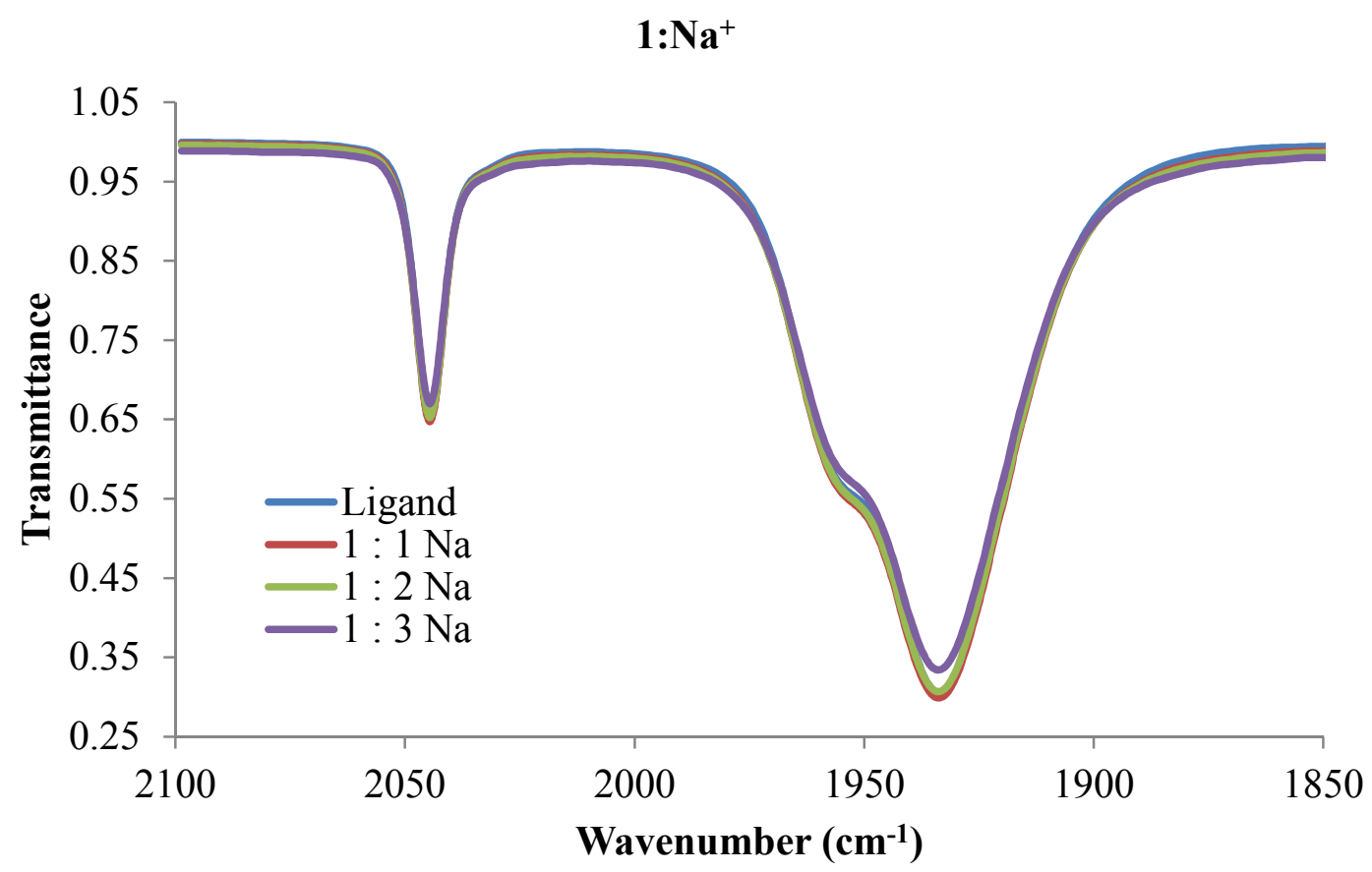

Figure S2. FTIR titration of 1 with NaBArF. 


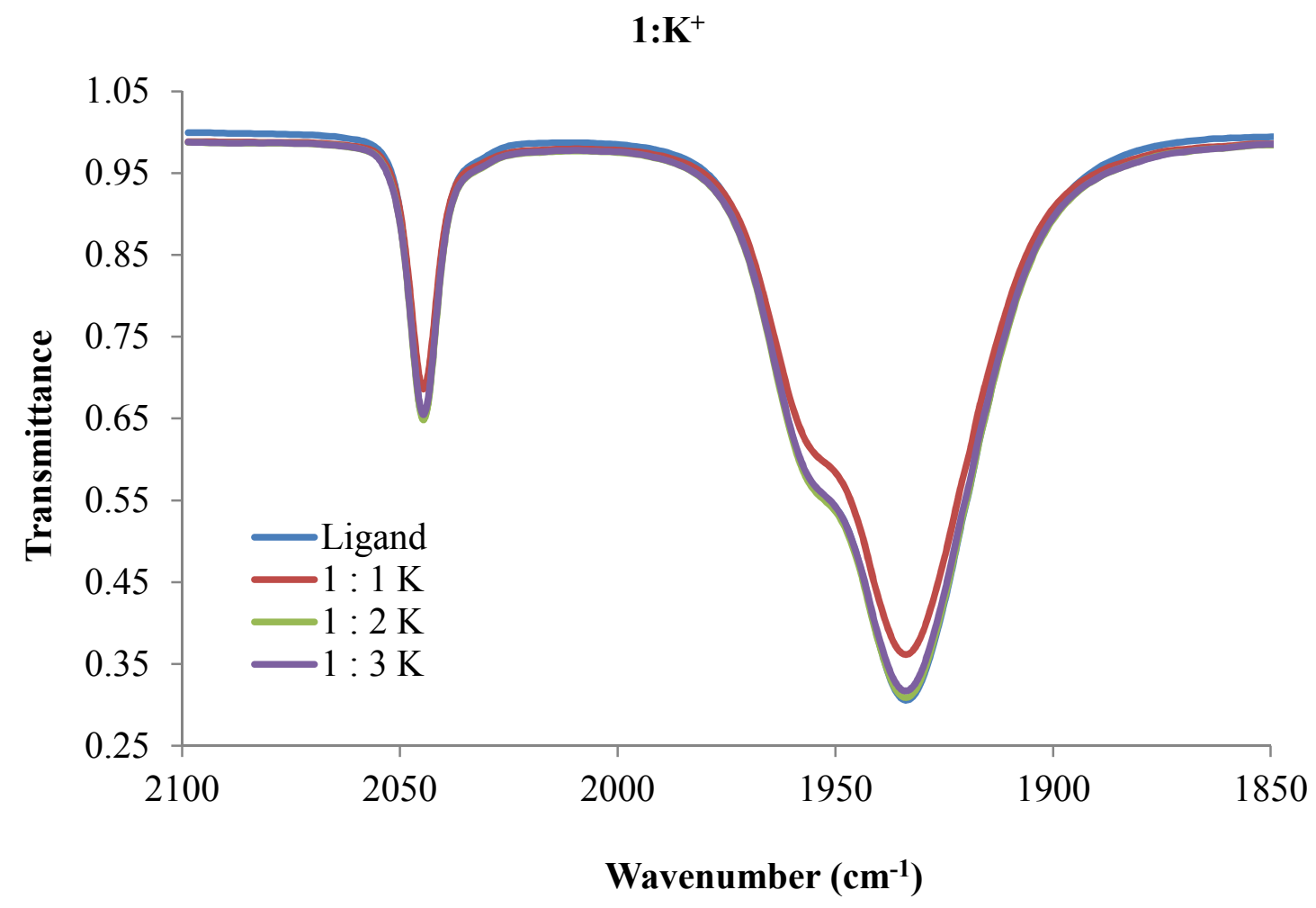

Figure S3. FTIR titration of 1 with $\mathrm{KB}\left(\mathrm{C}_{6} \mathrm{~F}_{5}\right)_{4}$.

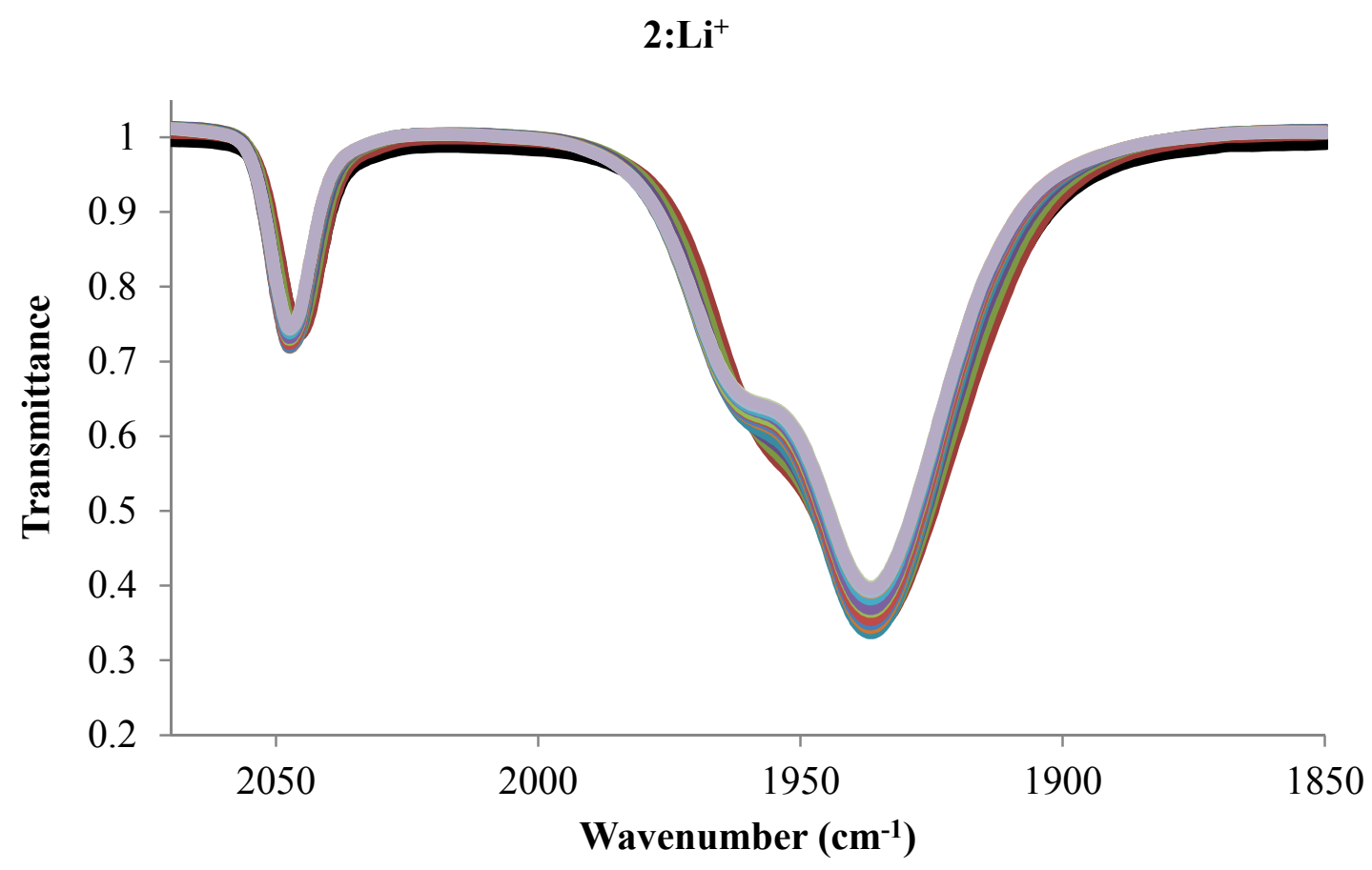

Figure S4. FTIR titration of 2 with $\mathrm{LiBPh}_{4} \cdot 3 \mathrm{dme}$. 


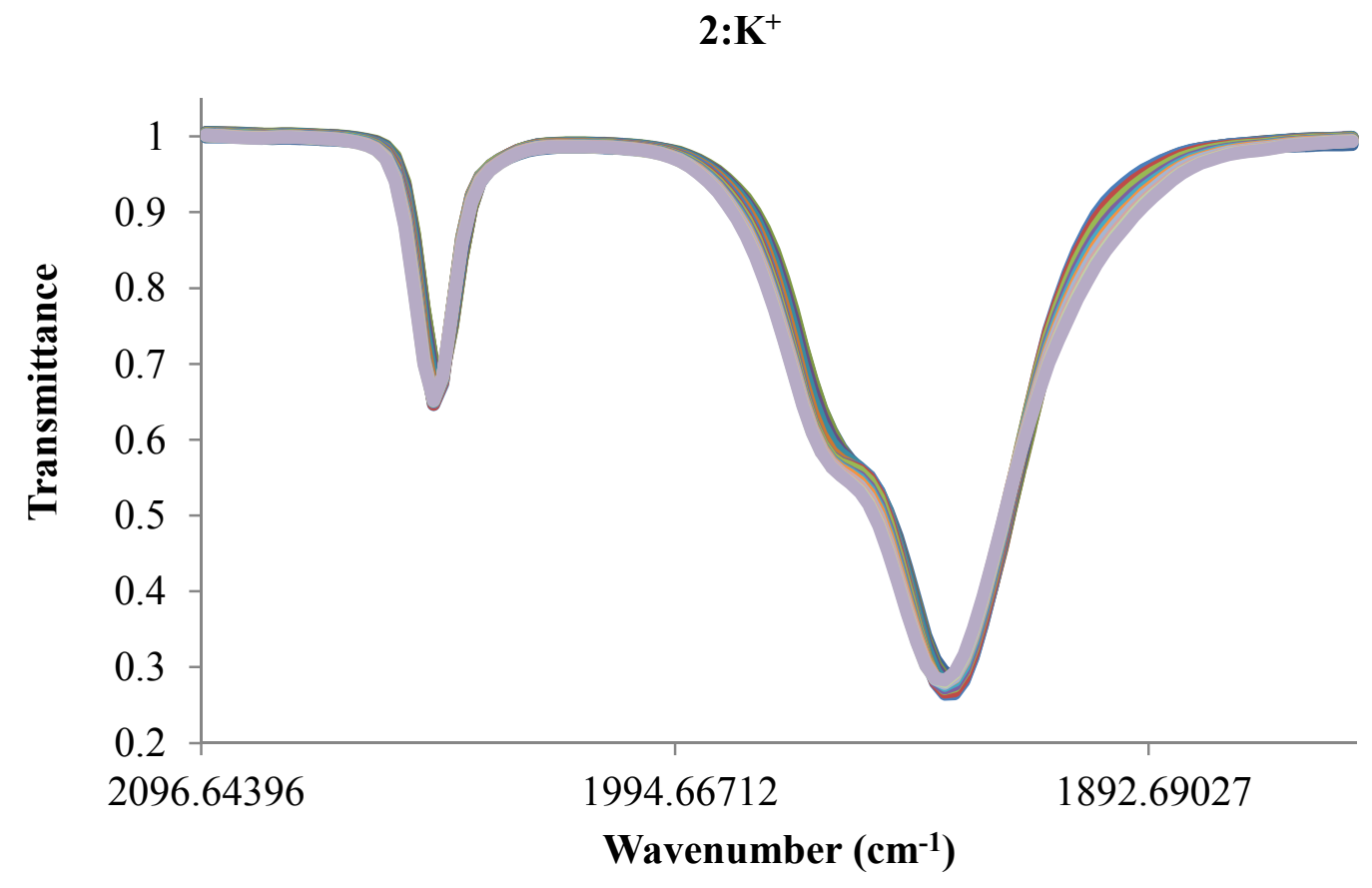

Figure S5. FTIR titration of 2 with $\mathrm{KB}\left(\mathrm{C}_{6} \mathrm{~F}_{5}\right)_{4}$.

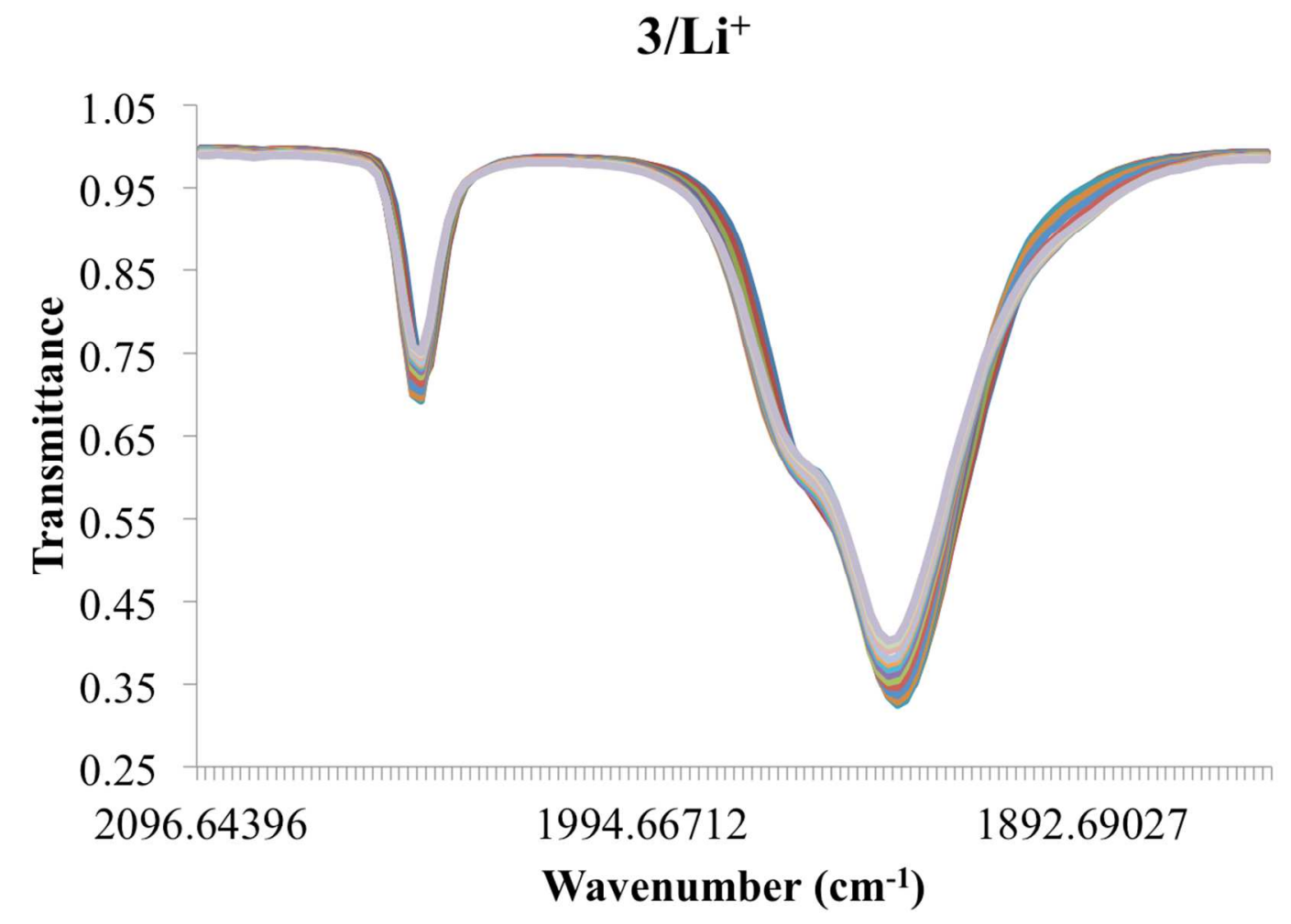

Figure S6. FTIR titration of 3 with $\mathrm{LiBPh}_{4} \cdot 3 \mathrm{dme}$. 


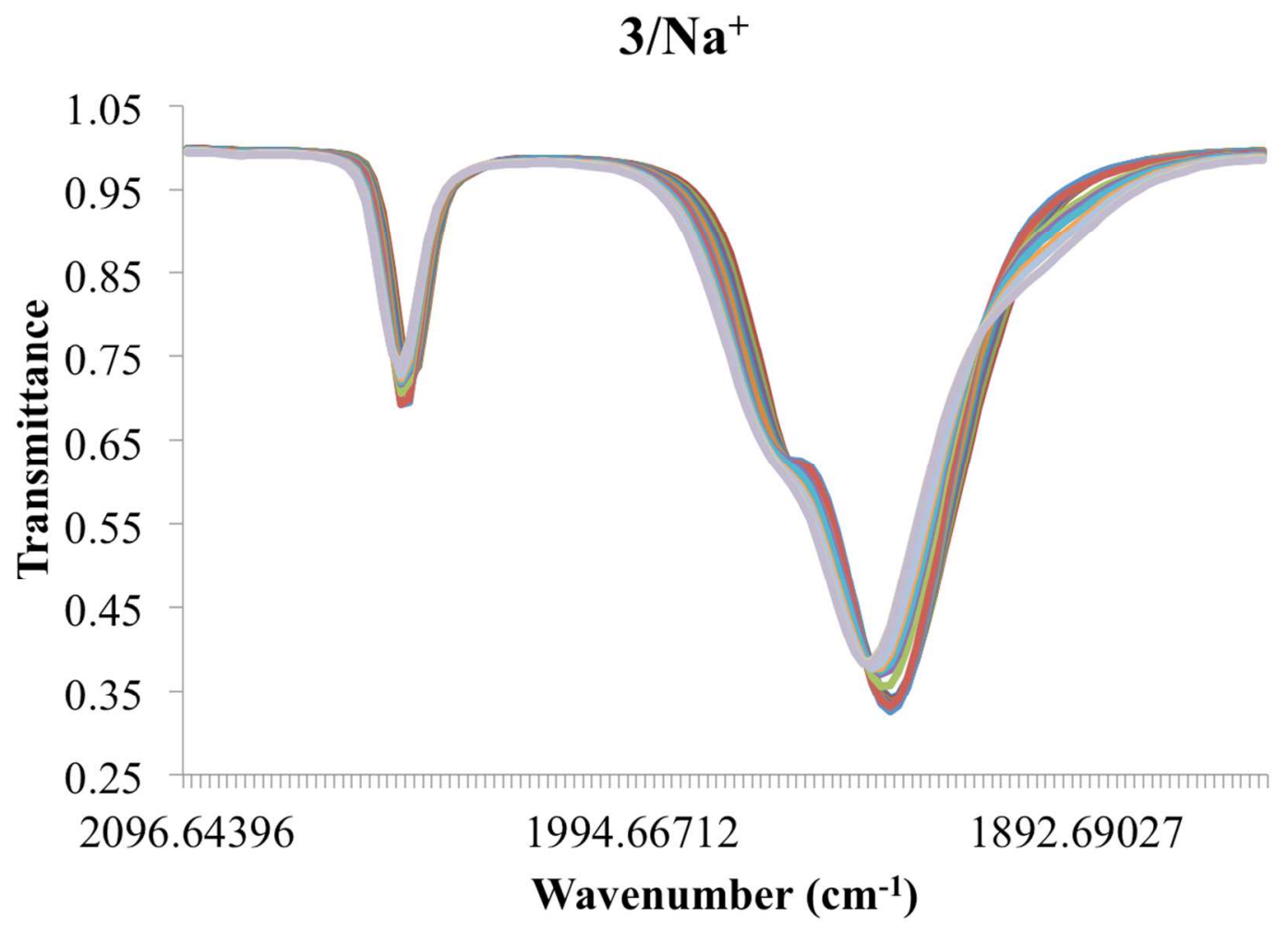

Figure S7. FTIR titration of $\mathbf{3}$ with $\mathrm{NaBArF}$.

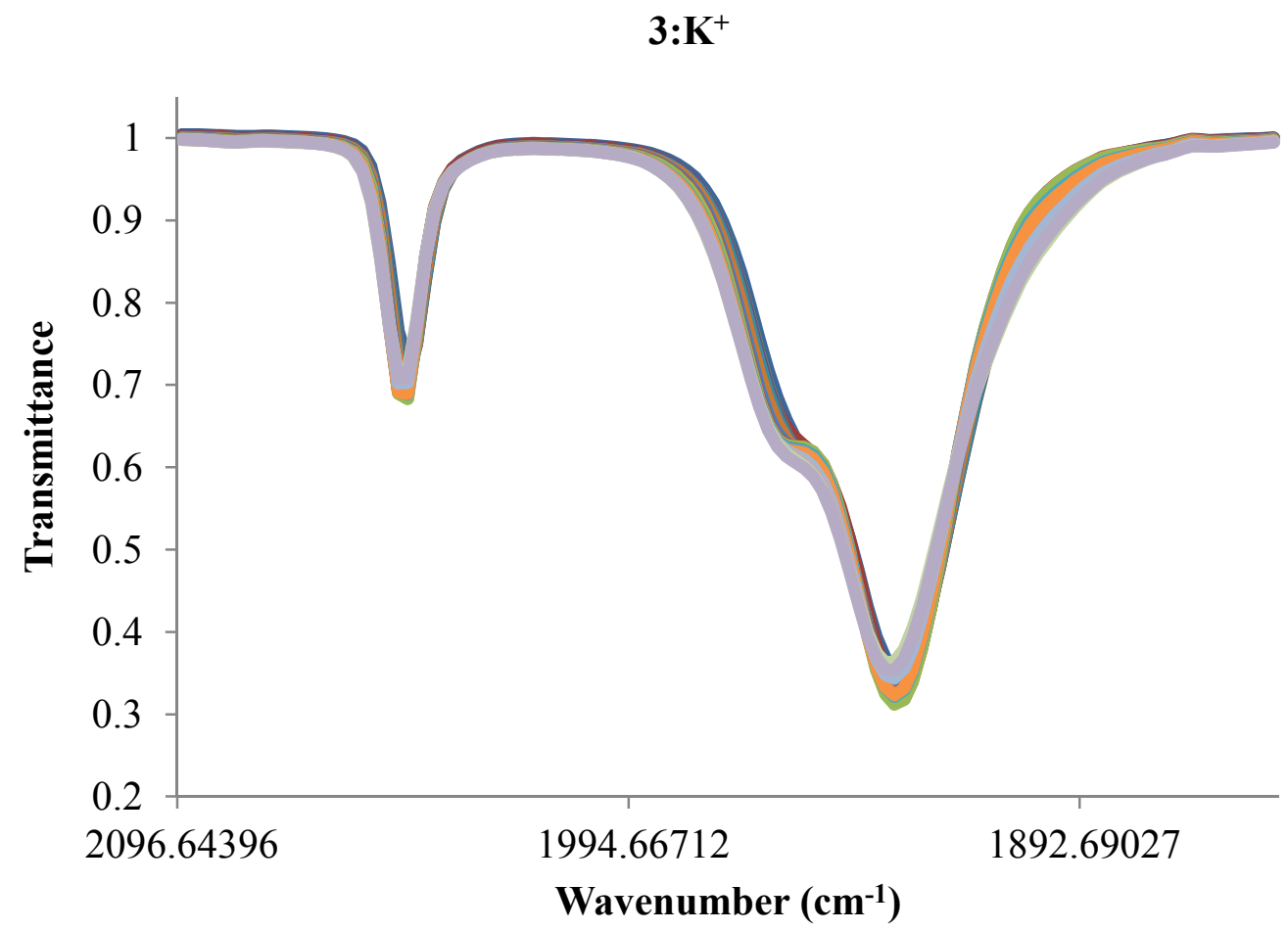

Figure S8. FTIR titration of 3 with $\mathrm{KB}\left(\mathrm{C}_{6} \mathrm{~F}_{5}\right)_{4}$. 
(C) NMR spectra of new ligands and complexes.
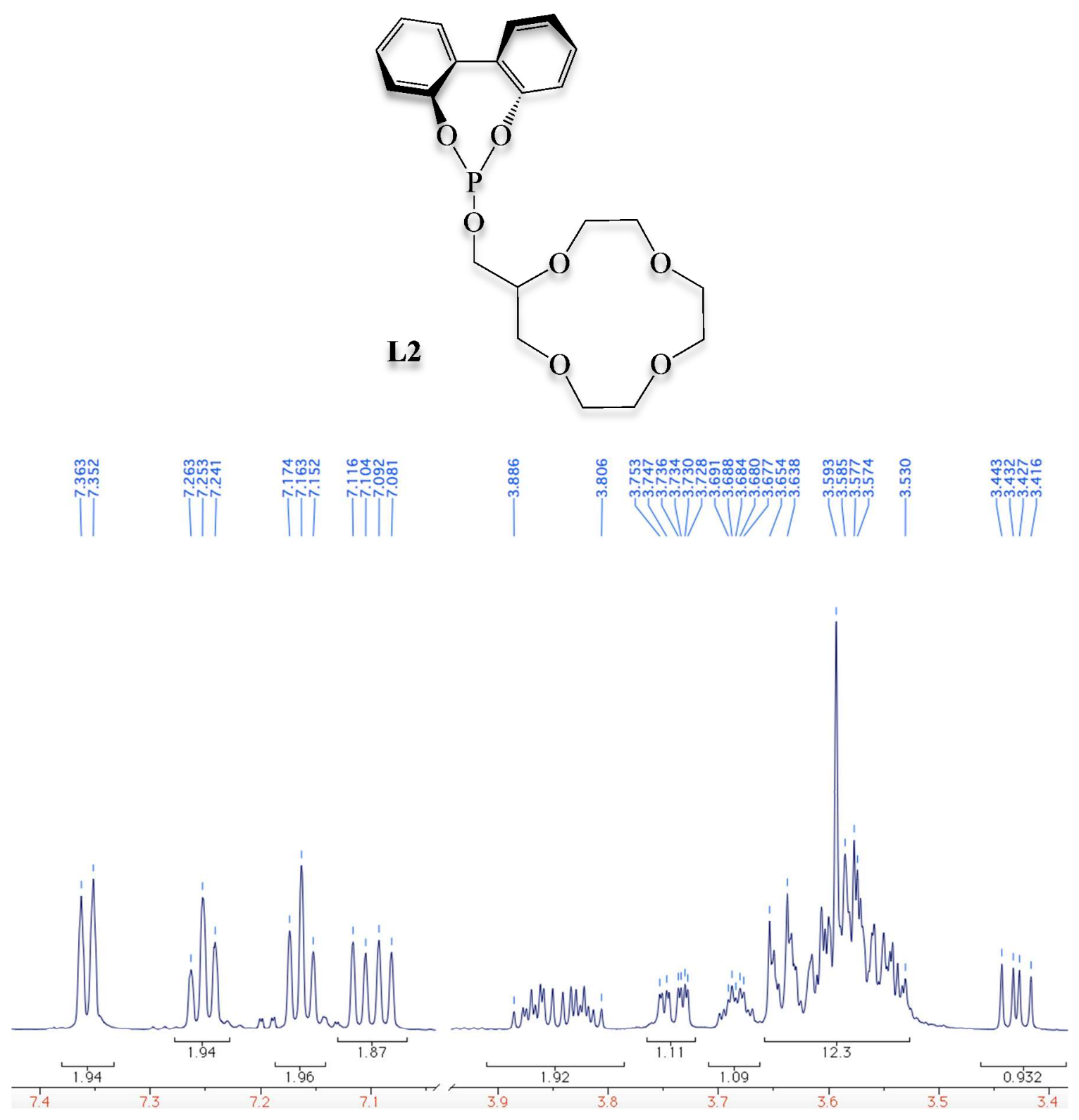

Figure S9. ${ }^{1} \mathrm{H}$ NMR spectrum $\left(700 \mathrm{MHz}, \mathrm{CDCl}_{3}\right.$ ) for ligand $\mathbf{L 2}$. (Some of the spectrum has been removed for clarity). 


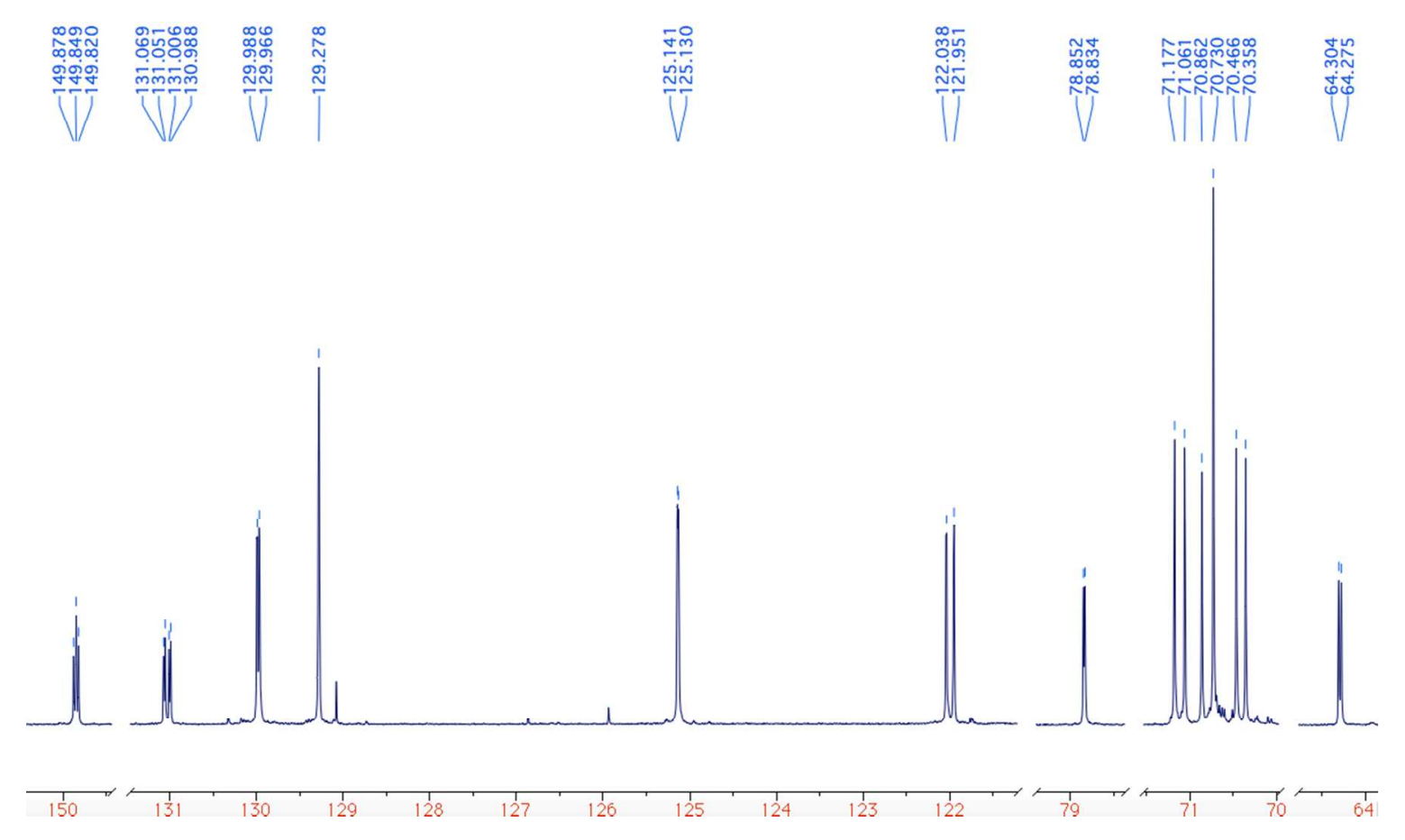

Figure S10. ${ }^{13} \mathrm{C}\left\{{ }^{1} \mathrm{H}\right\}$ NMR spectrum $\left(700 \mathrm{MHz}, \mathrm{CDCl}_{3}\right)$ for ligand $\mathbf{L 2}$. (Some of the spectrum has been removed for clarity).

$$
\frac{\frac{6}{d}}{\frac{5}{\sigma}}
$$

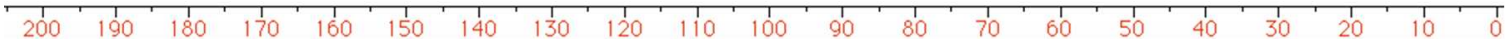

Figure S11. ${ }^{31} \mathrm{P}\left\{{ }^{1} \mathrm{H}\right\} \mathrm{NMR}$ spectrum $\left(400 \mathrm{MHz}, \mathrm{CDCl}_{3}\right)$ for ligand $\mathbf{L}$. 


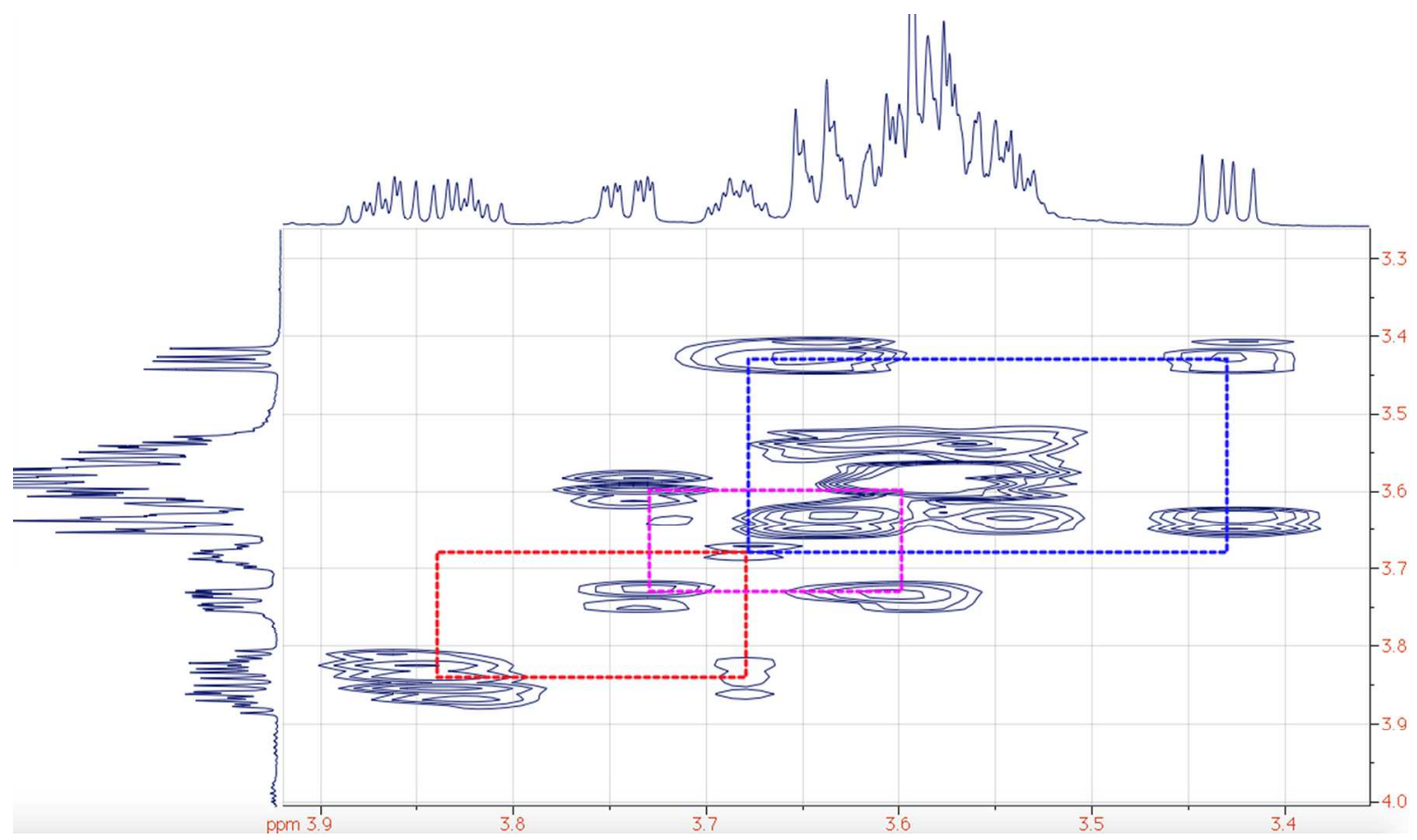

Figure S12. Aliphatic portion of ${ }^{1} \mathrm{H}-{ }^{1} \mathrm{H}$ COSY spectrum $\left(700 \mathrm{MHz}, \mathrm{CDCl}_{3}\right)$ for ligand L2.

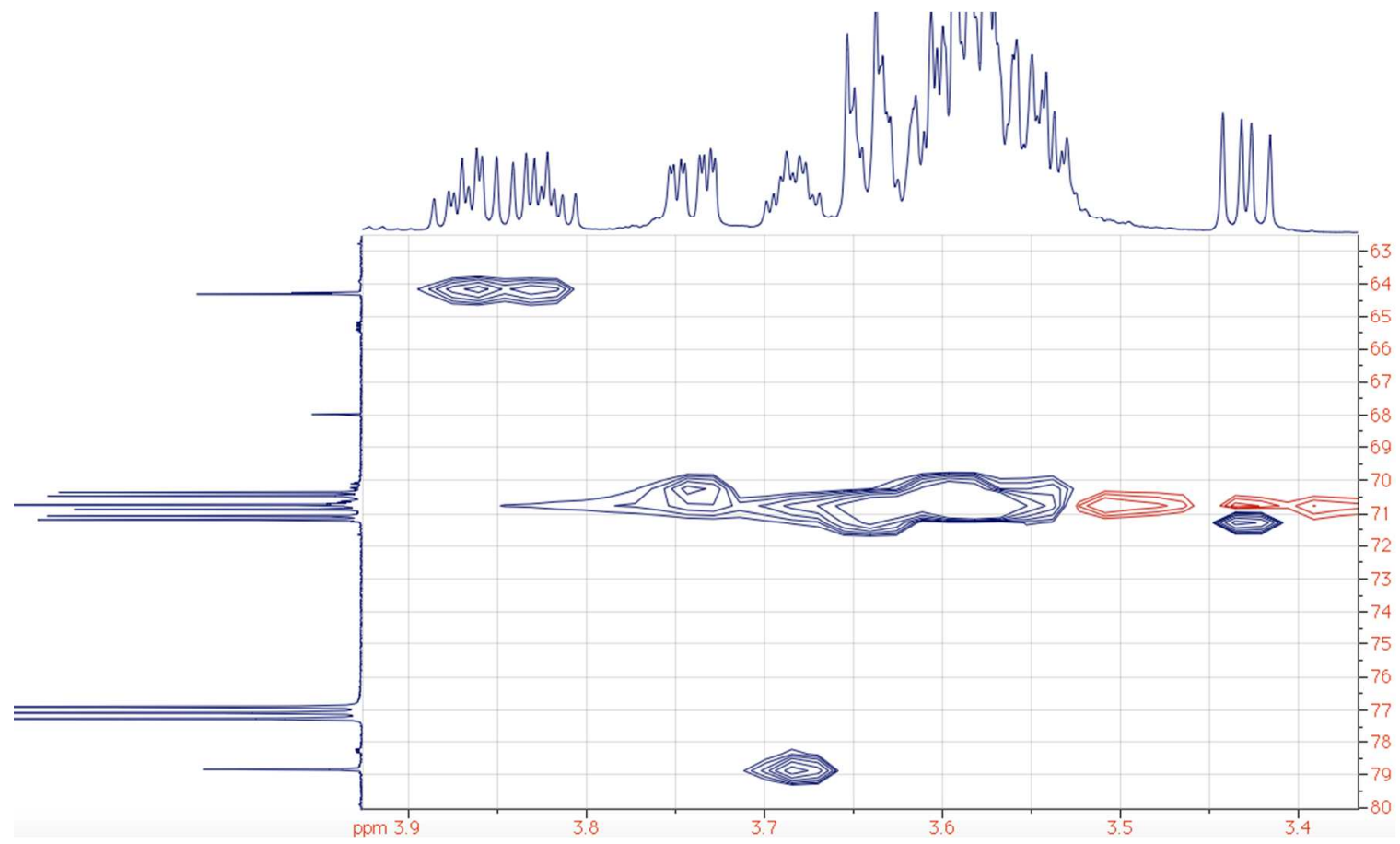

Figure S13. Aliphatic portion of ${ }^{13} \mathrm{C}-{ }^{1} \mathrm{H}$ HSQC spectrum $\left(700 \mathrm{MHz}, \mathrm{CDCl}_{3}\right)$ for ligand L2. 

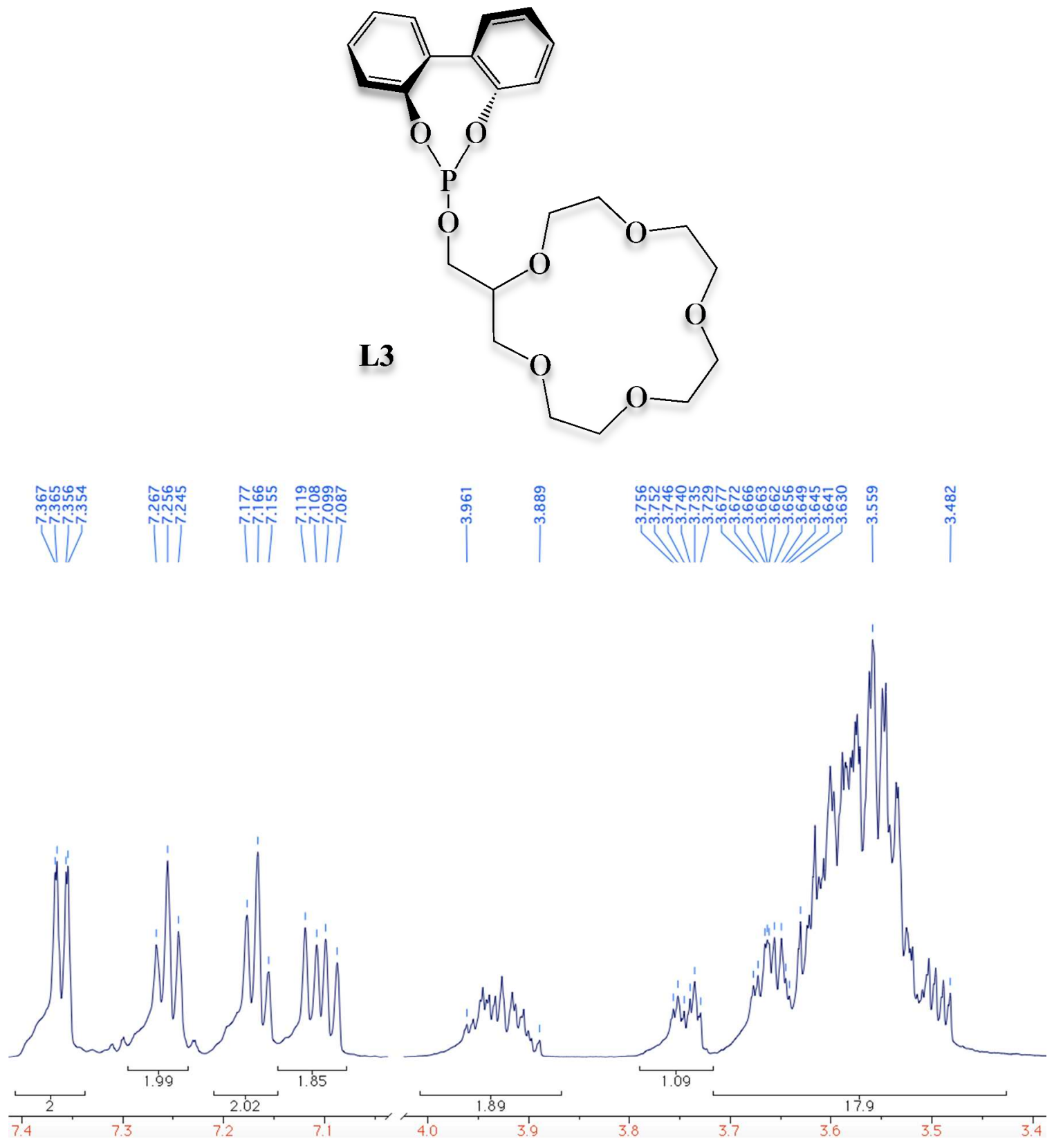

Figure S14. ${ }^{1} \mathrm{H}$ NMR spectrum $\left(700 \mathrm{MHz}, \mathrm{CDCl}_{3}\right.$ ) for ligand $\mathbf{L 3}$. (Some of the spectrum has been removed for clarity). 


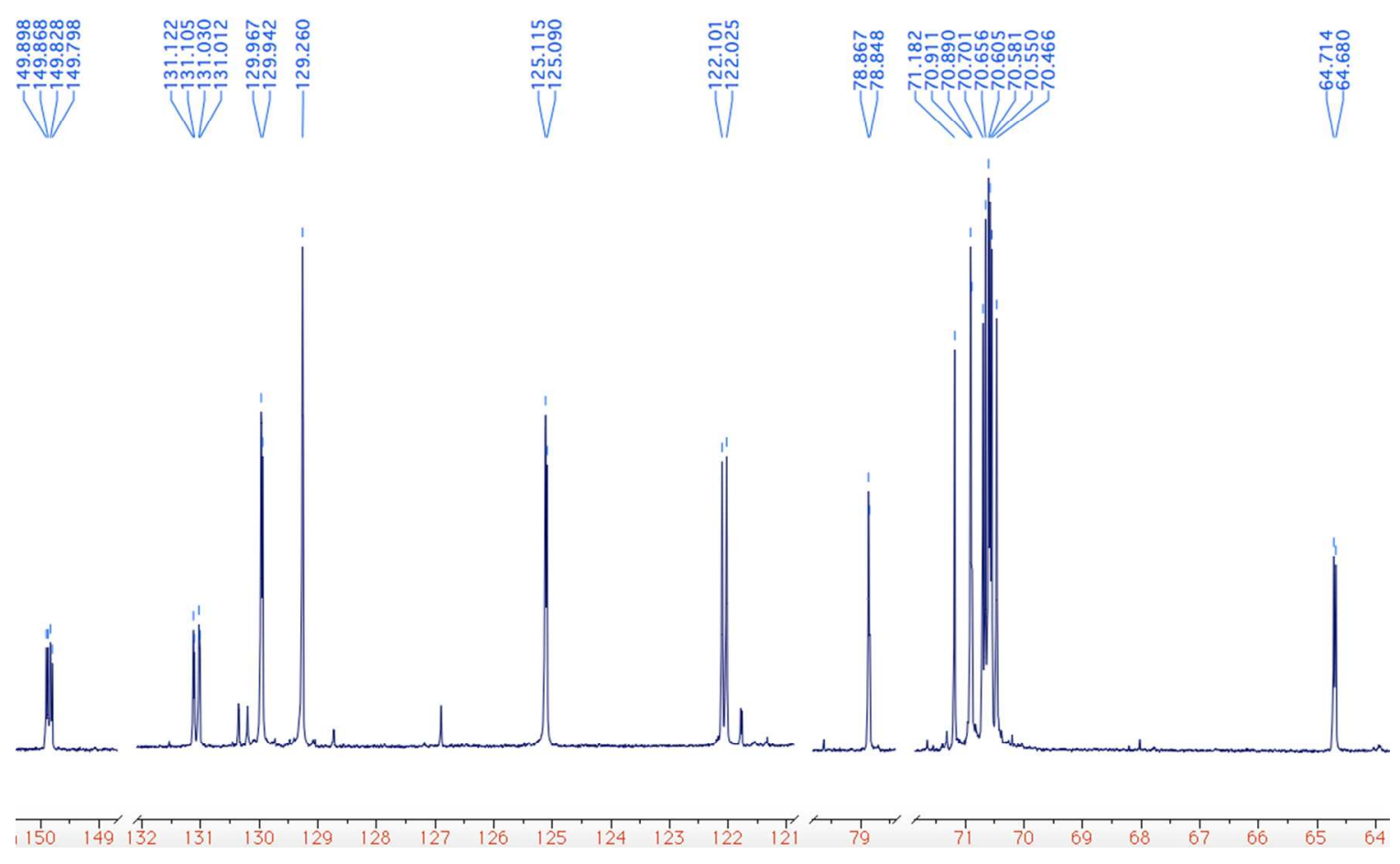

Figure S15. ${ }^{13} \mathrm{C}\left\{{ }^{1} \mathrm{H}\right\}$ NMR spectrum $\left(700 \mathrm{MHz}, \mathrm{CDCl}_{3}\right)$ for ligand $\mathbf{L 3}$. (Some of the spectrum has been removed for clarity).
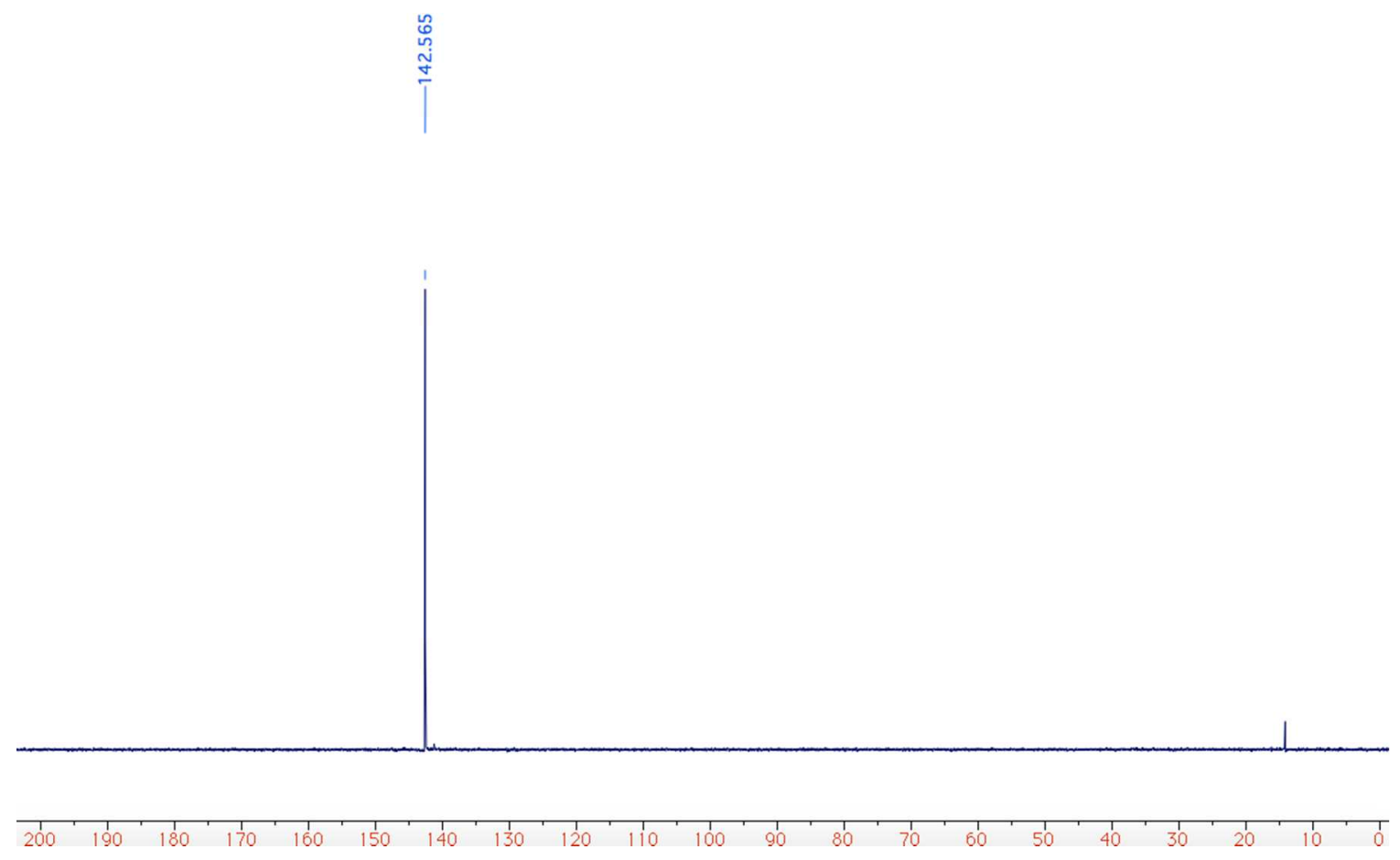

Figure S16. ${ }^{31} \mathrm{P}\left\{{ }^{1} \mathrm{H}\right\}$ NMR spectrum $\left(400 \mathrm{MHz}, \mathrm{CDCl}_{3}\right)$ for ligand $\mathbf{L 3}$. 

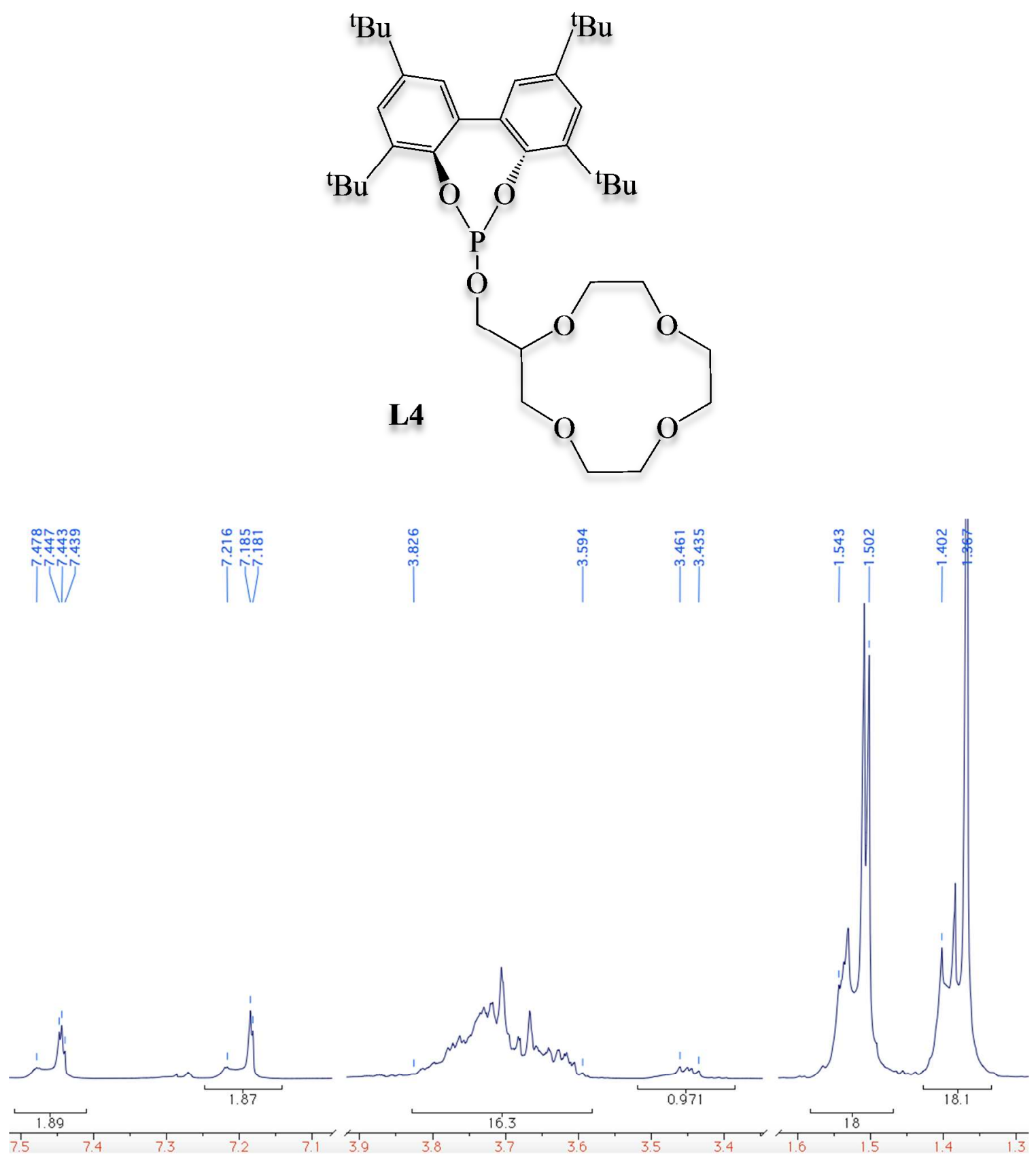

Figure S17. ${ }^{1} \mathrm{H}$ NMR spectrum $\left(700 \mathrm{MHz}, \mathrm{CDCl}_{3}\right.$ ) for ligand $\mathbf{L 4}$. (Some of the spectrum has been removed for clarity). 


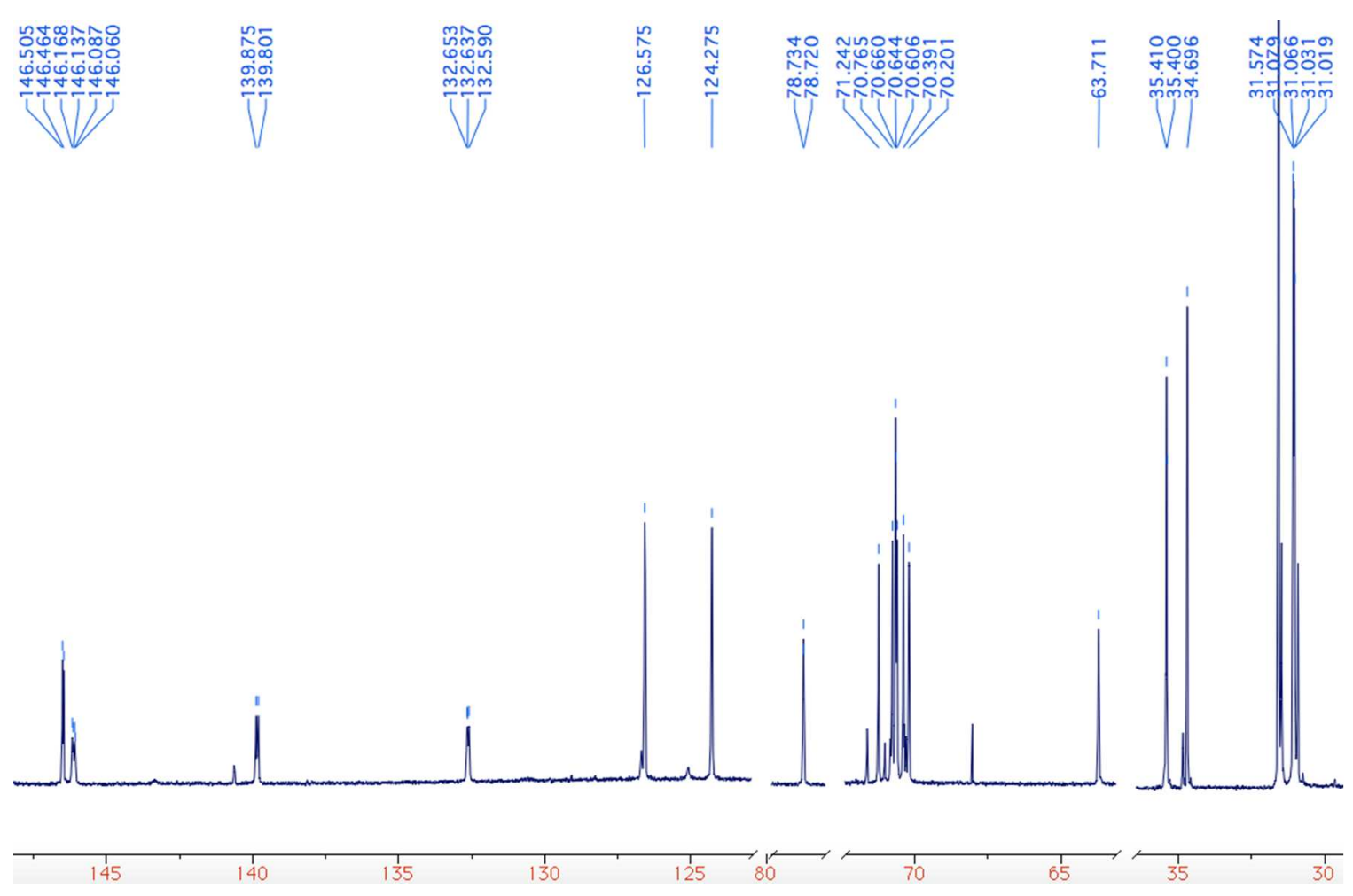

Figure S18. ${ }^{13} \mathrm{C}\left\{{ }^{1} \mathrm{H}\right\}$ NMR spectrum $\left(700 \mathrm{MHz}, \mathrm{CDCl}_{3}\right)$ for ligand $\mathbf{L 4}$. (Some of the spectrum has been removed for clarity).
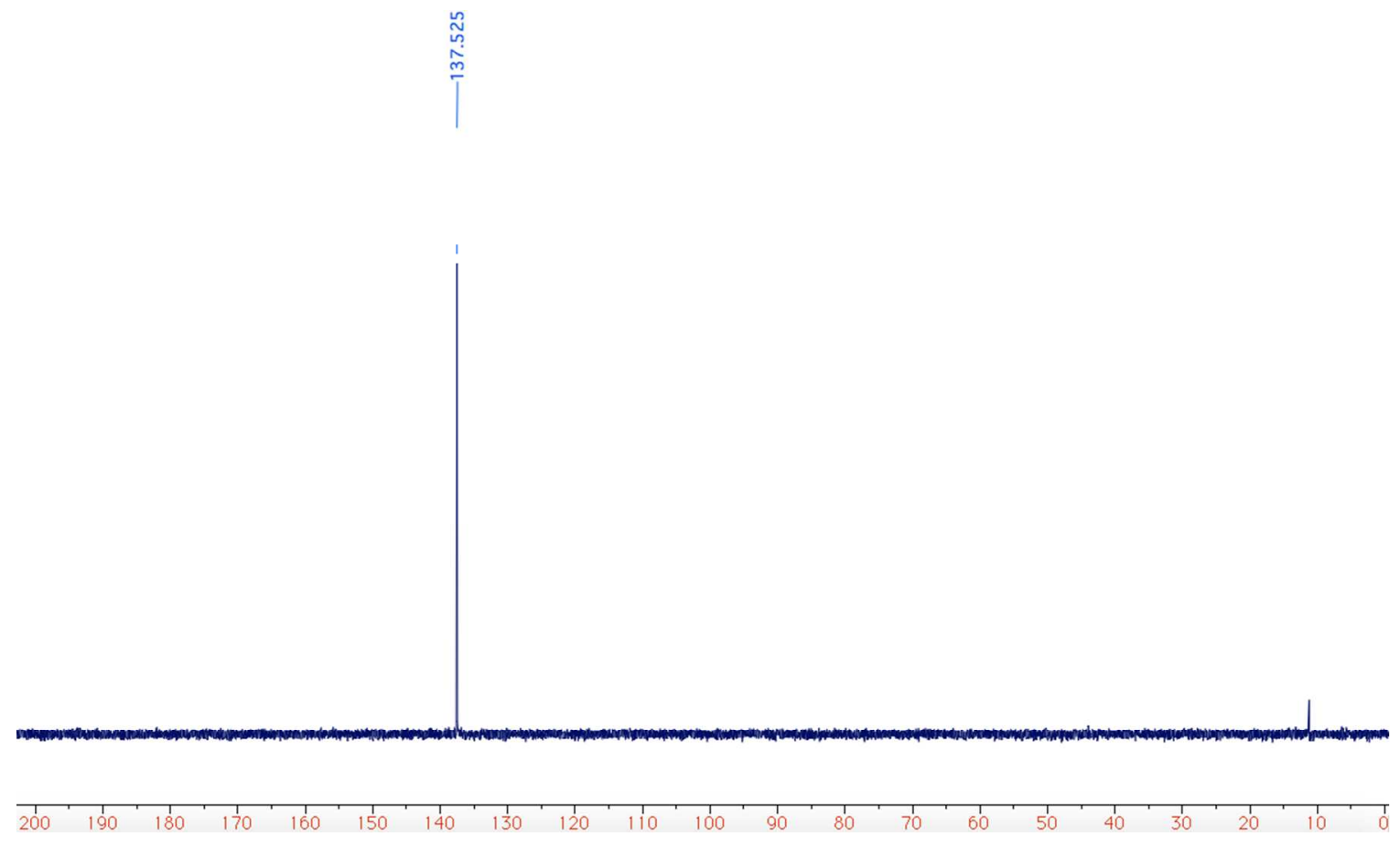

Figure S19. ${ }^{31} \mathrm{P}\left\{{ }^{1} \mathrm{H}\right\}$ NMR spectrum $\left(400 \mathrm{MHz}, \mathrm{CDCl}_{3}\right)$ for ligand $\mathbf{L 4}$. 

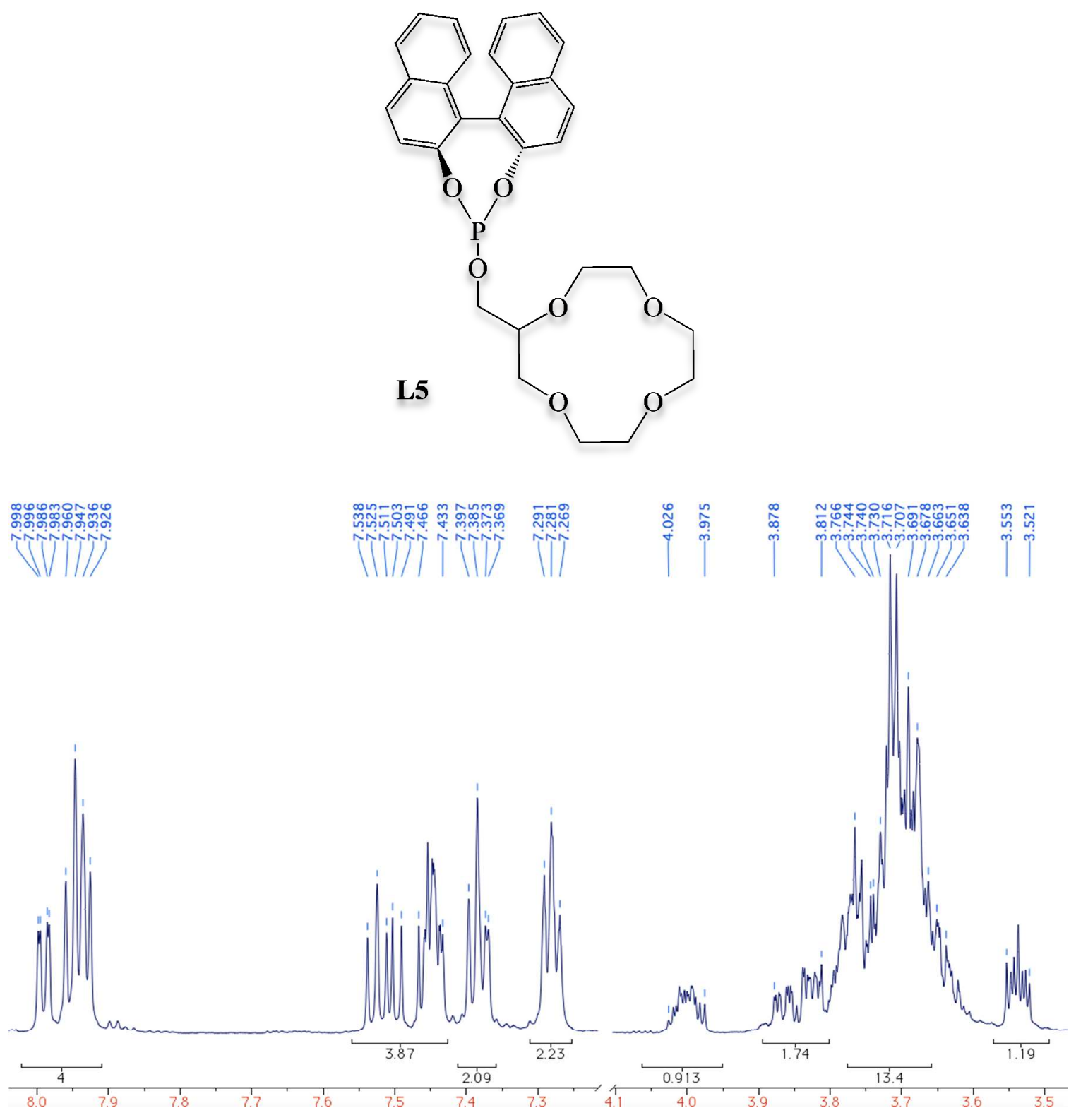

Figure S20. ${ }^{1} \mathrm{H}$ NMR spectrum $\left(700 \mathrm{MHz}, \mathrm{CDCl}_{3}\right.$ ) for ligand $\mathbf{L 5}$. (Some of the spectrum has been removed for clarity). 

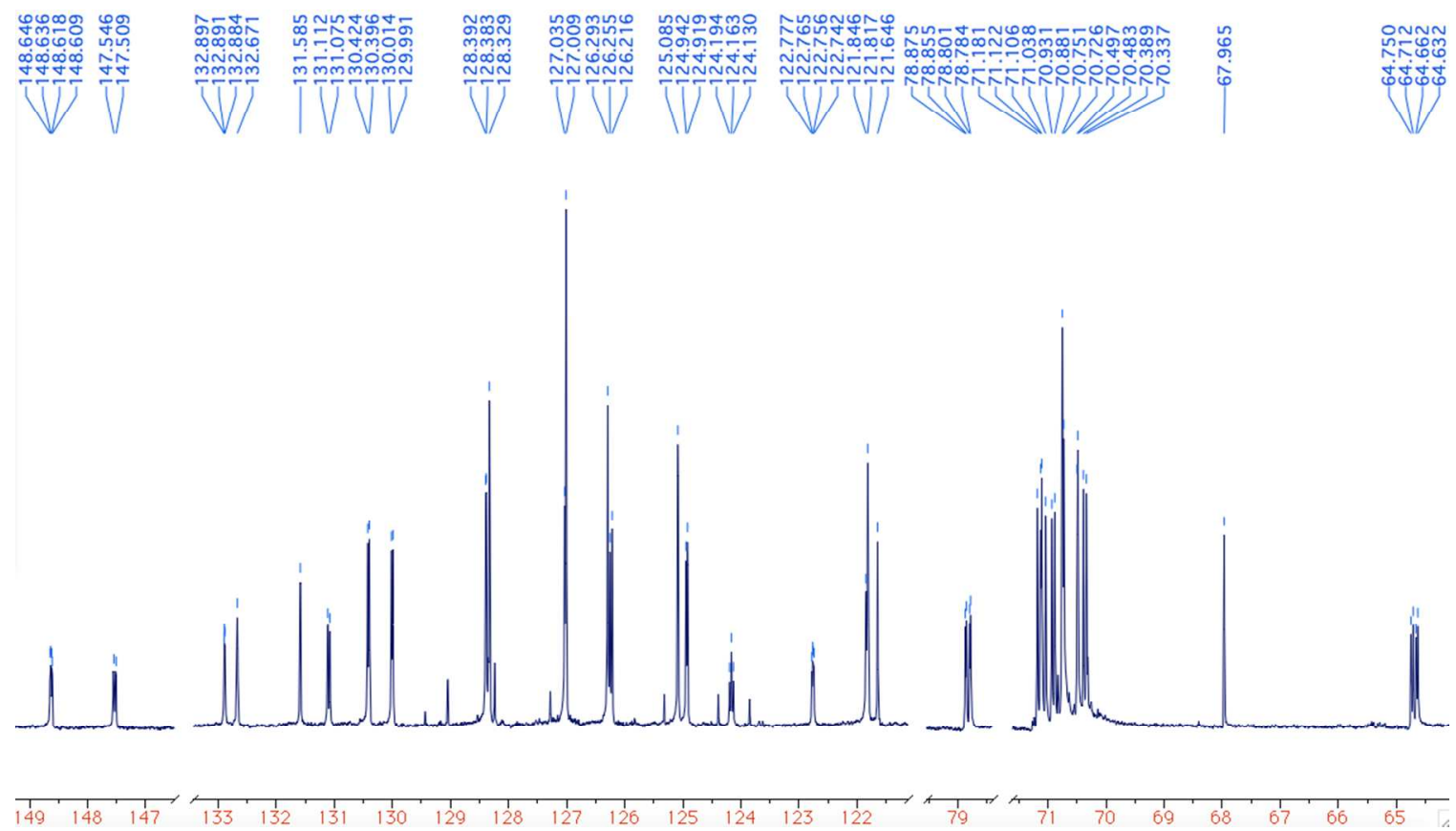

Figure S21. ${ }^{13} \mathrm{C}\left\{{ }^{1} \mathrm{H}\right\}$ NMR spectrum $\left(700 \mathrm{MHz}, \mathrm{CDCl}_{3}\right)$ for ligand $\mathbf{L 5}$. (Some of the spectrum has been removed for clarity).
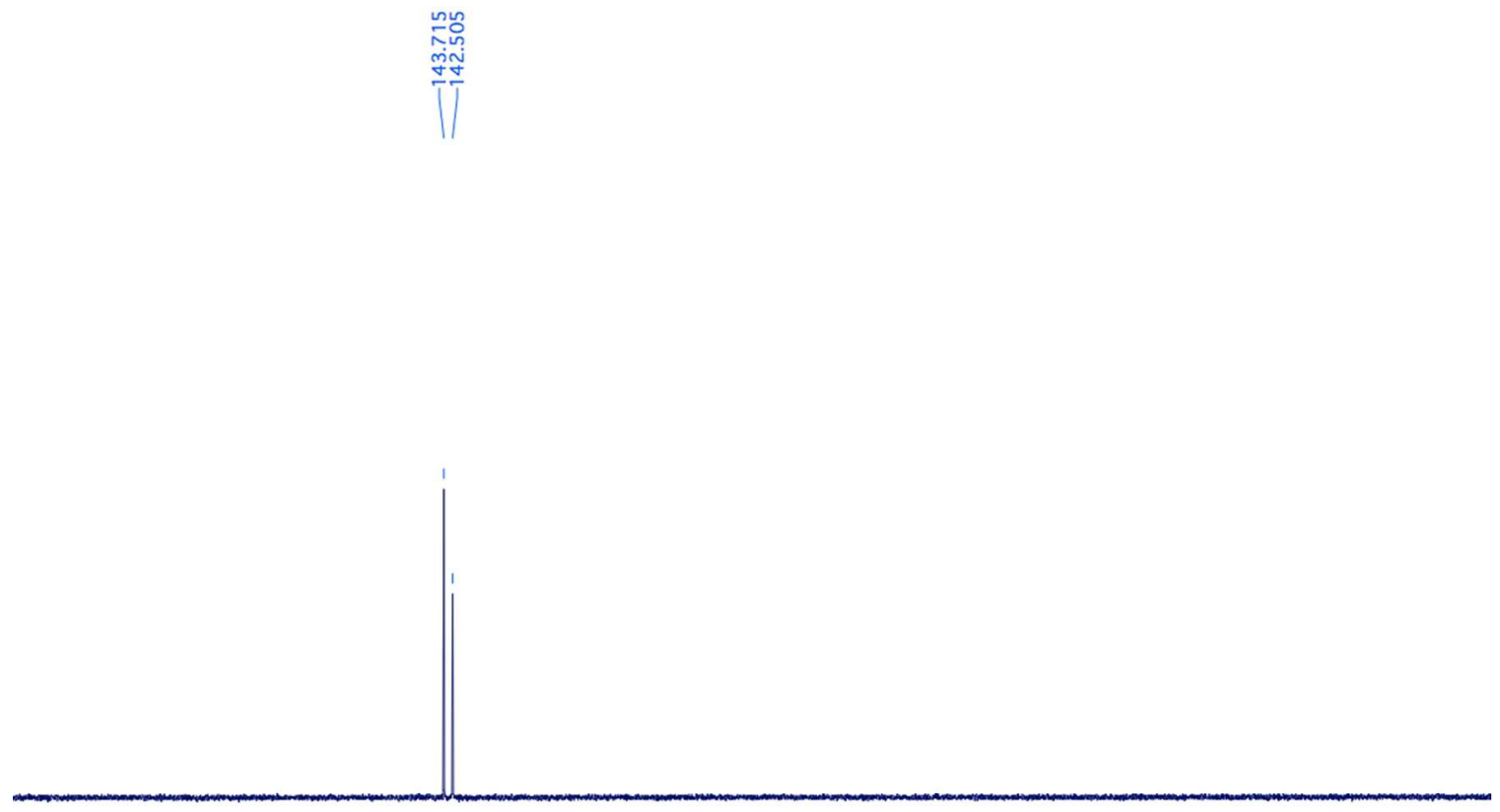

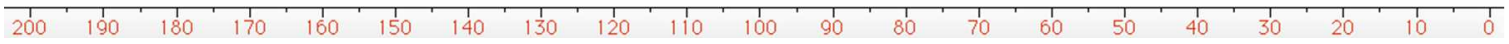

Figure S22. ${ }^{31} \mathrm{P}\left\{{ }^{1} \mathrm{H}\right\} \mathrm{NMR}$ spectrum $\left(400 \mathrm{MHz}, \mathrm{CDCl}_{3}\right)$ for ligand $\mathbf{L 5}$. 


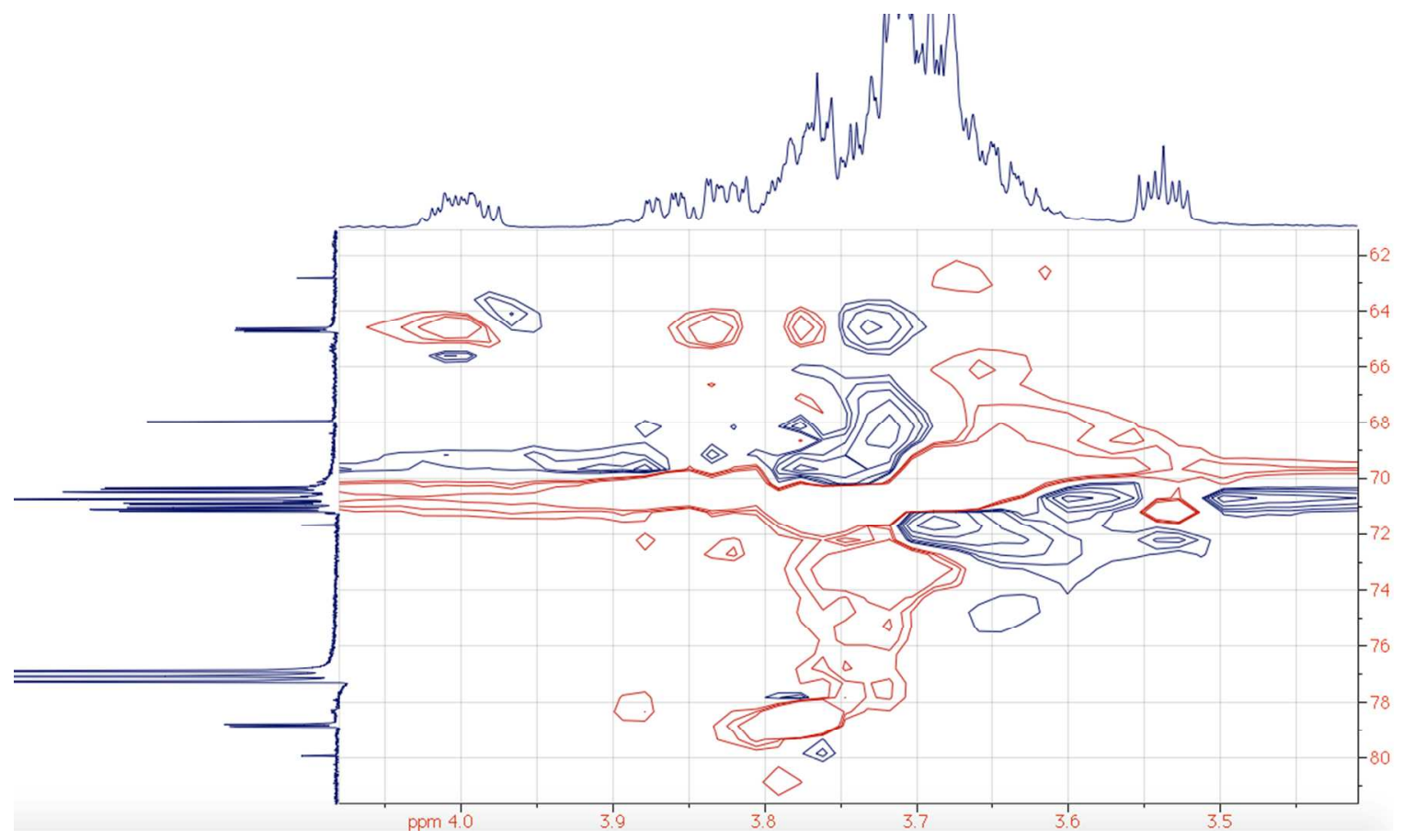

Figure S23. Aliphatic portion of ${ }^{13} \mathrm{C}-{ }^{1} \mathrm{H}$ HSQC spectrum (700 $\mathrm{MHz}, \mathrm{CDCl}_{3}$ ) for ligand L5. 

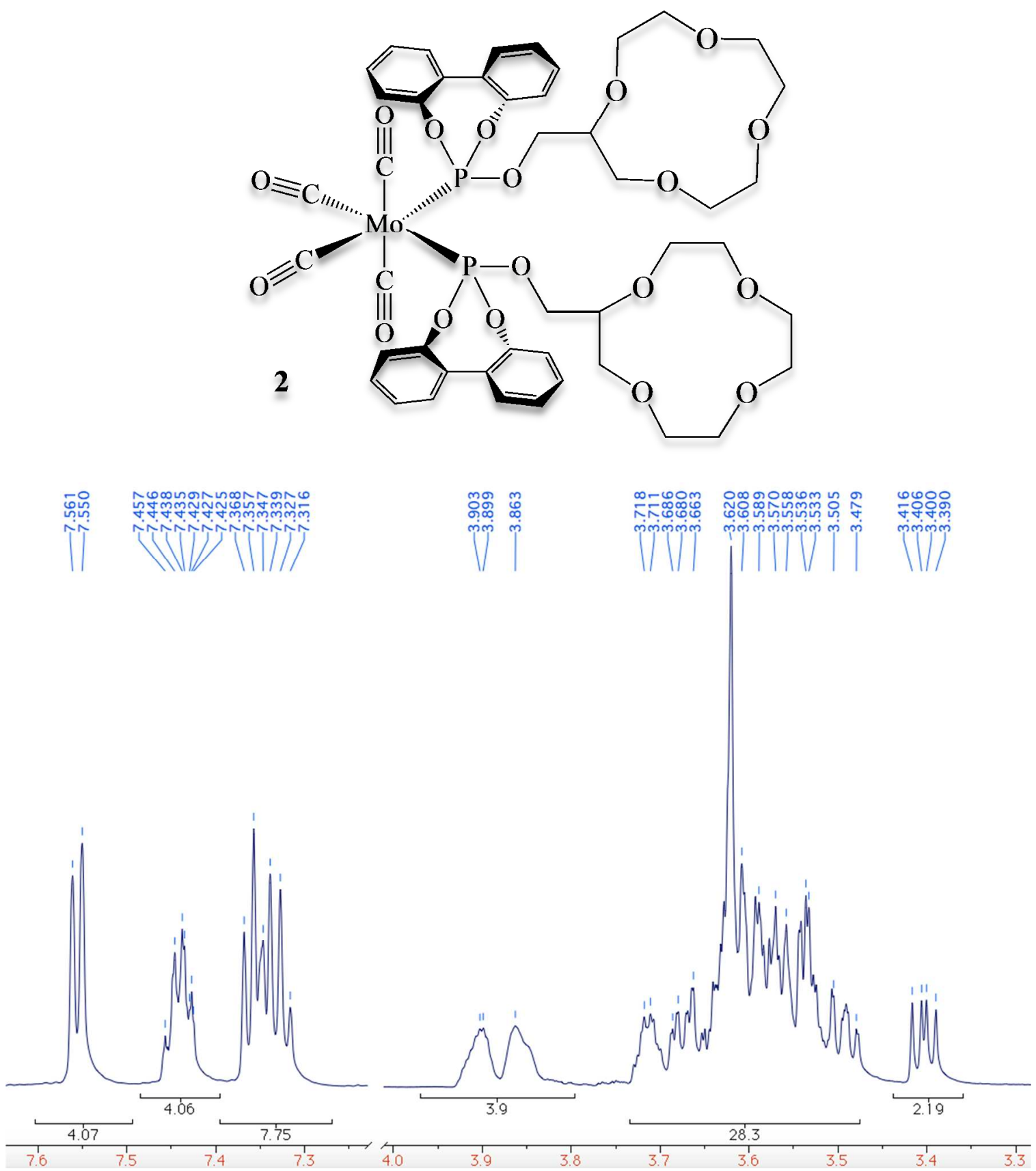

Figure S24. ${ }^{1} \mathrm{H} \mathrm{NMR}$ spectrum $\left(700 \mathrm{MHz}, \mathrm{CD}_{2} \mathrm{Cl}_{2}\right.$ ) for complex 2. (Some of the spectrum has been removed for clarity). 


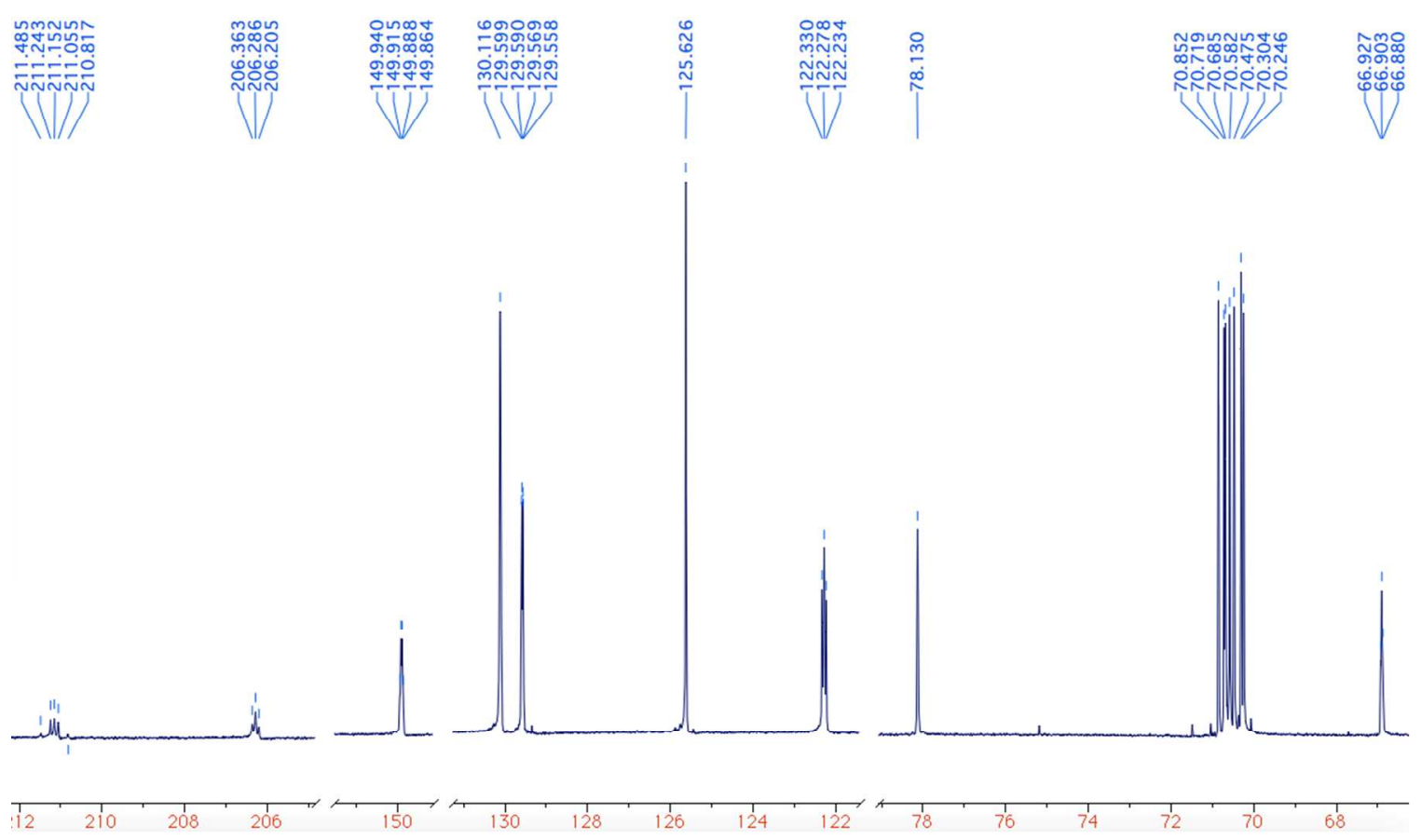

Figure S25. ${ }^{13} \mathrm{C}\left\{{ }^{1} \mathrm{H}\right\}$ NMR spectrum $\left(700 \mathrm{MHz}, \mathrm{CD}_{2} \mathrm{Cl}_{2}\right)$ for complex 2. (Some of the spectrum has been removed for clarity).
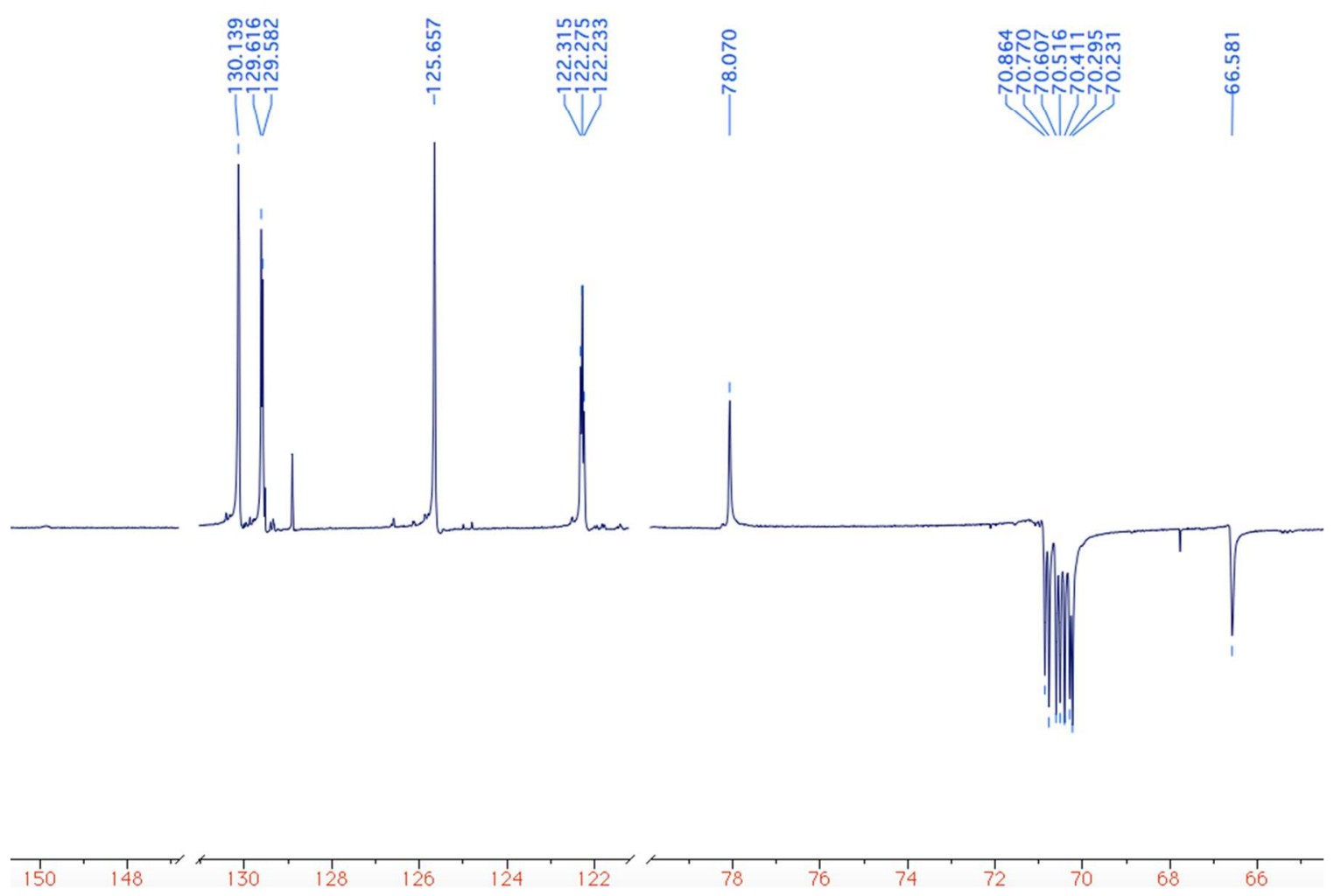

Figure S26. DEPT135 NMR spectrum (700 MHz, $\mathrm{CD}_{2} \mathrm{Cl}_{2}$ ) for complex 2. (Some of the spectrum has been removed for clarity). 

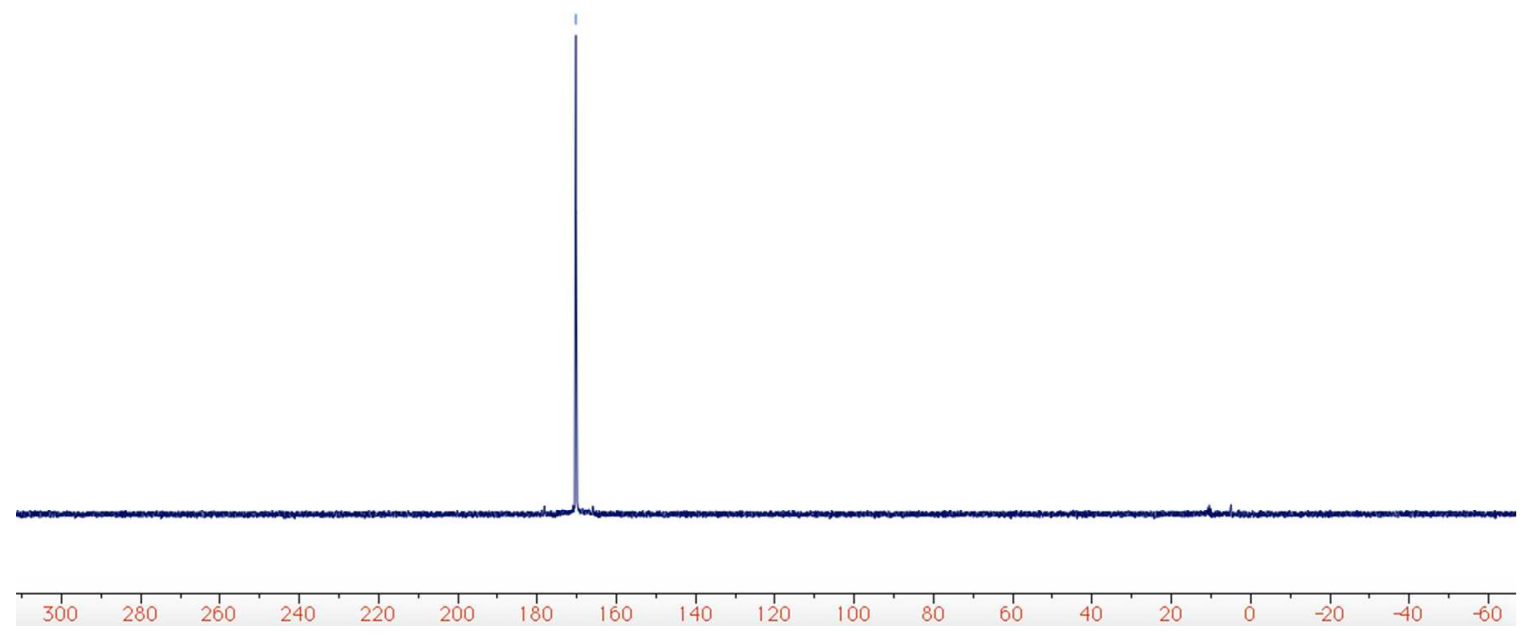

Figure S27. ${ }^{31} \mathrm{P}\left\{{ }^{1} \mathrm{H}\right\}$ NMR spectrum $\left(400 \mathrm{MHz}, \mathrm{CD}_{2} \mathrm{Cl}_{2}\right)$ for complex 2.

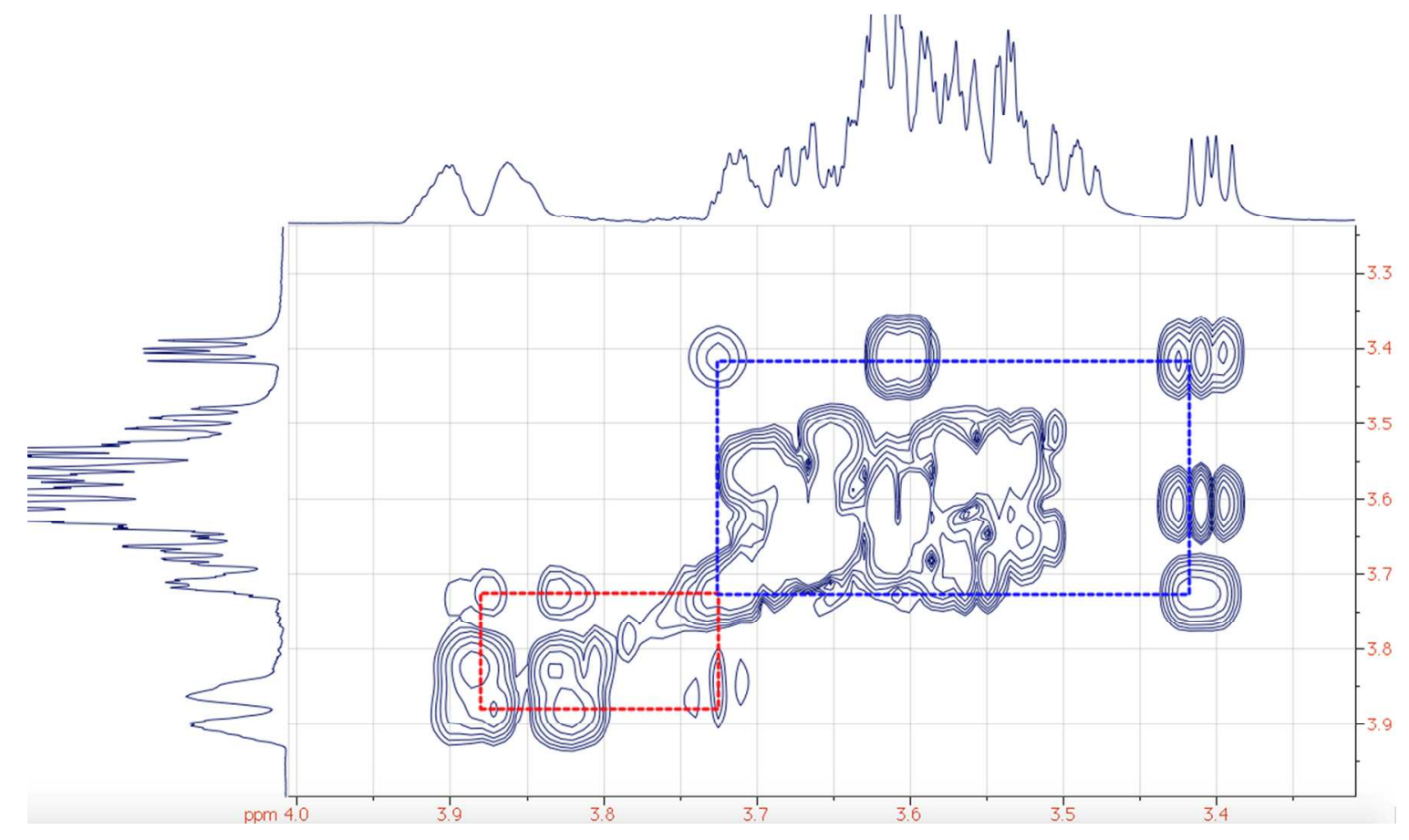

Figure S28. Aliphatic portion of ${ }^{1} \mathrm{H}-{ }^{1} \mathrm{H}$ COSY spectrum $\left(700 \mathrm{MHz}, \mathrm{CD}_{2} \mathrm{Cl}_{2}\right)$ for complex 2 . 


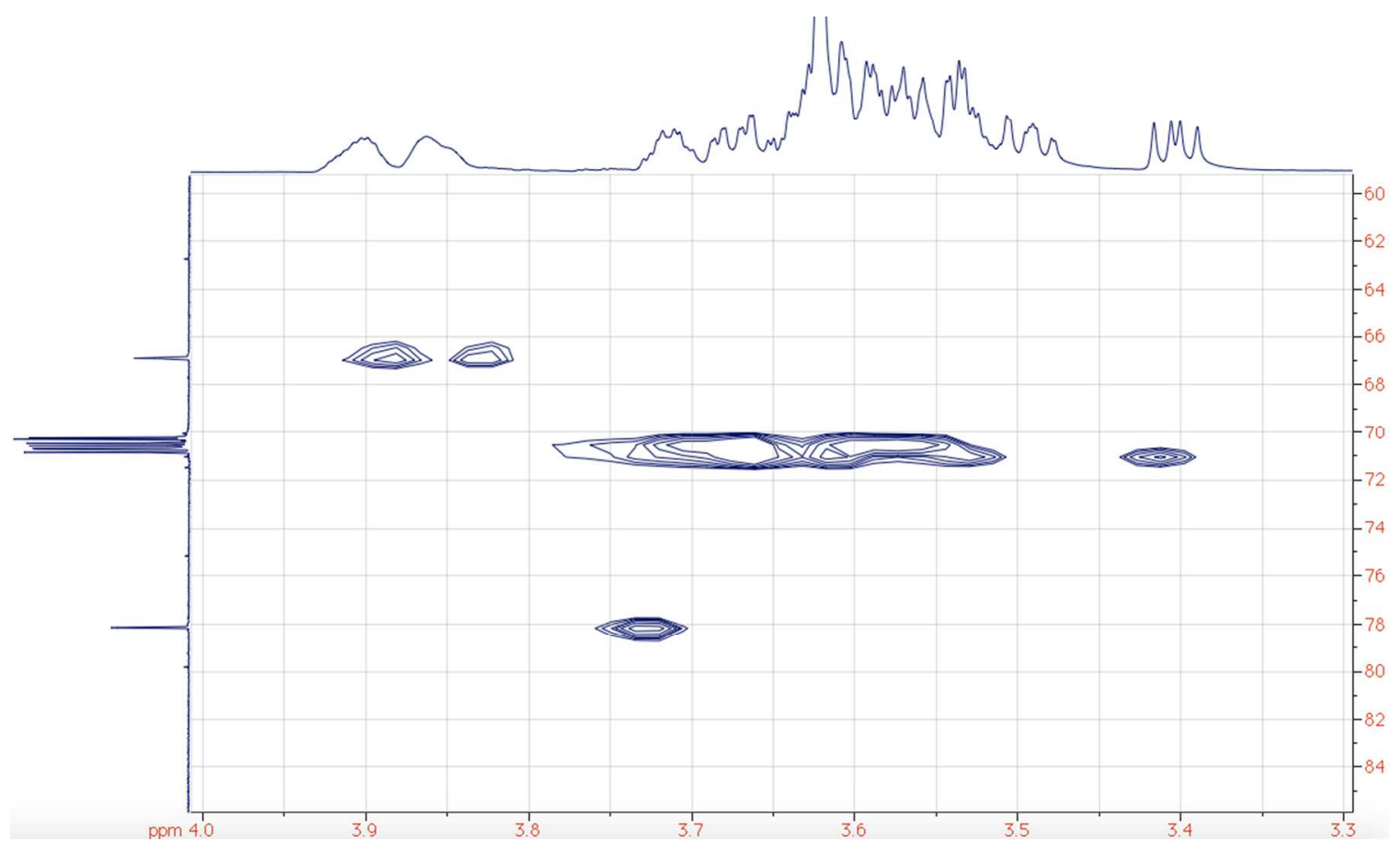

Figure S29. Aliphatic portion of ${ }^{13} \mathrm{C}-{ }^{1} \mathrm{H}$ HSQC spectrum $\left(700 \mathrm{MHz}, \mathrm{CD}_{2} \mathrm{Cl}_{2}\right)$ for complex 2. 

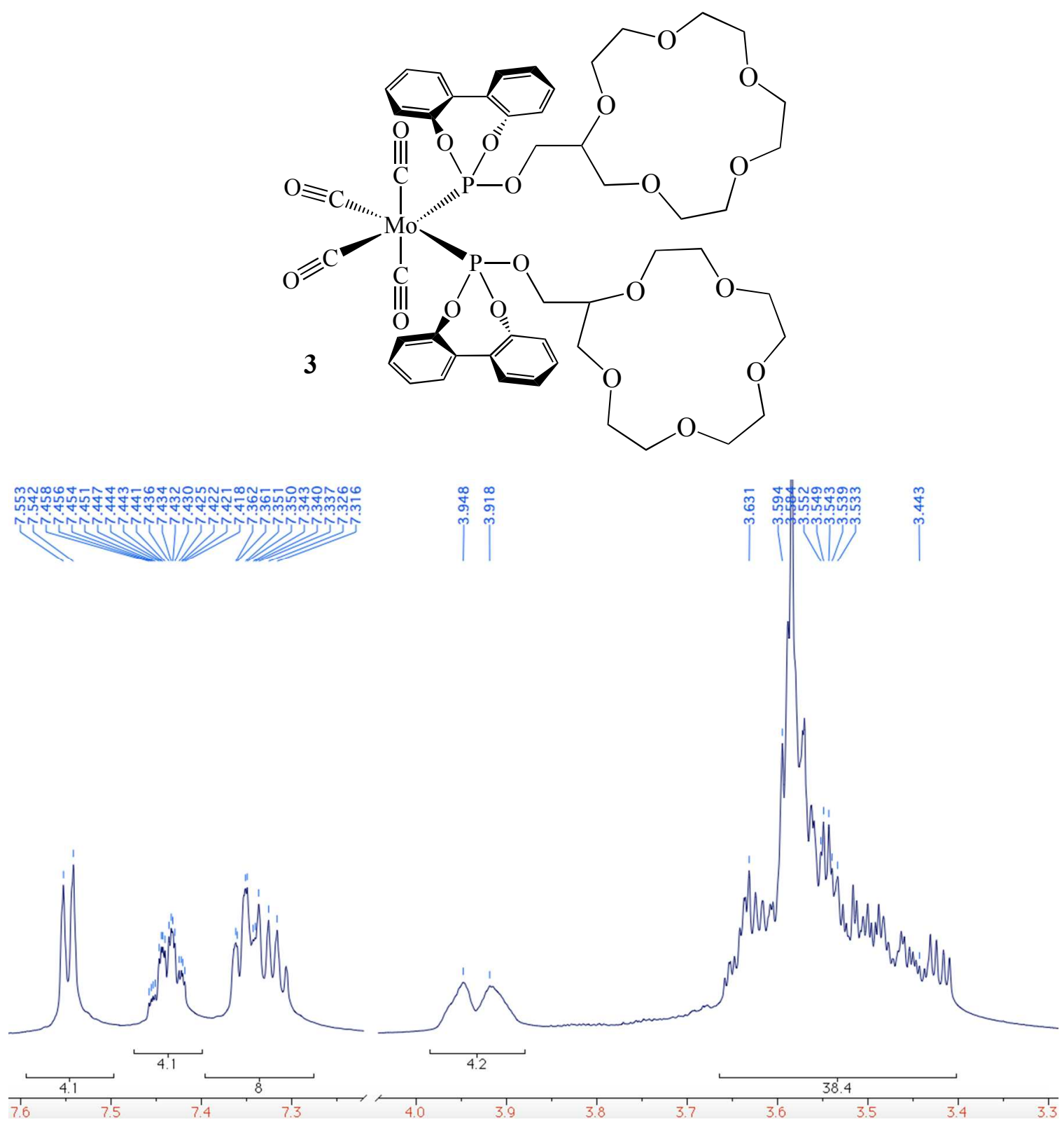

Figure S30. ${ }^{1} \mathrm{H}$ NMR spectrum $\left(700 \mathrm{MHz}, \mathrm{CD}_{2} \mathrm{Cl}_{2}\right.$ ) for complex 3. (Some of the spectrum has been removed for clarity). 


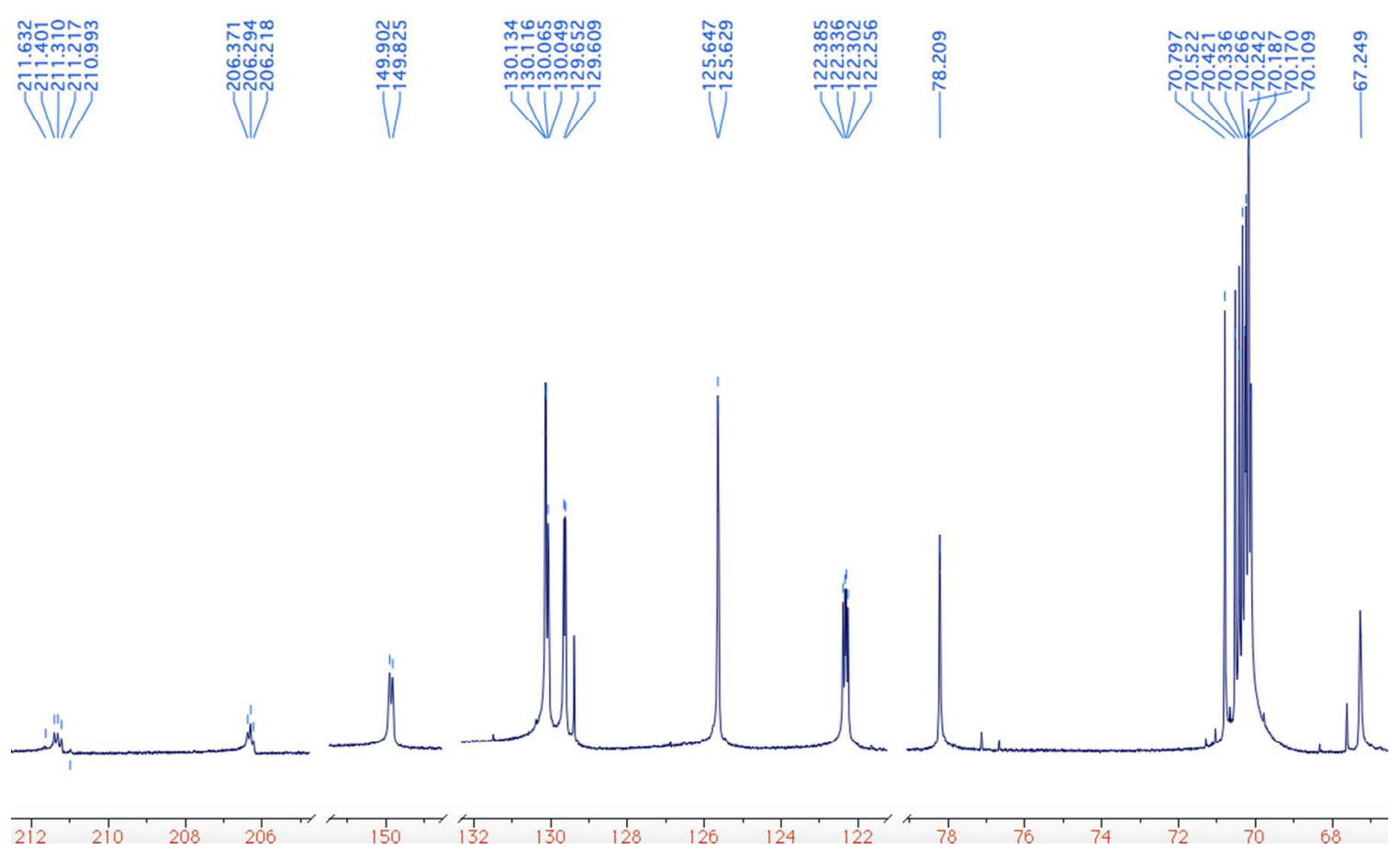

Figure S31. ${ }^{13} \mathrm{C}\left\{{ }^{1} \mathrm{H}\right\}$ NMR spectrum $\left(700 \mathrm{MHz}, \mathrm{CD}_{2} \mathrm{Cl}_{2}\right.$ ) for complex 3. (Some of the spectrum has been removed for clarity).
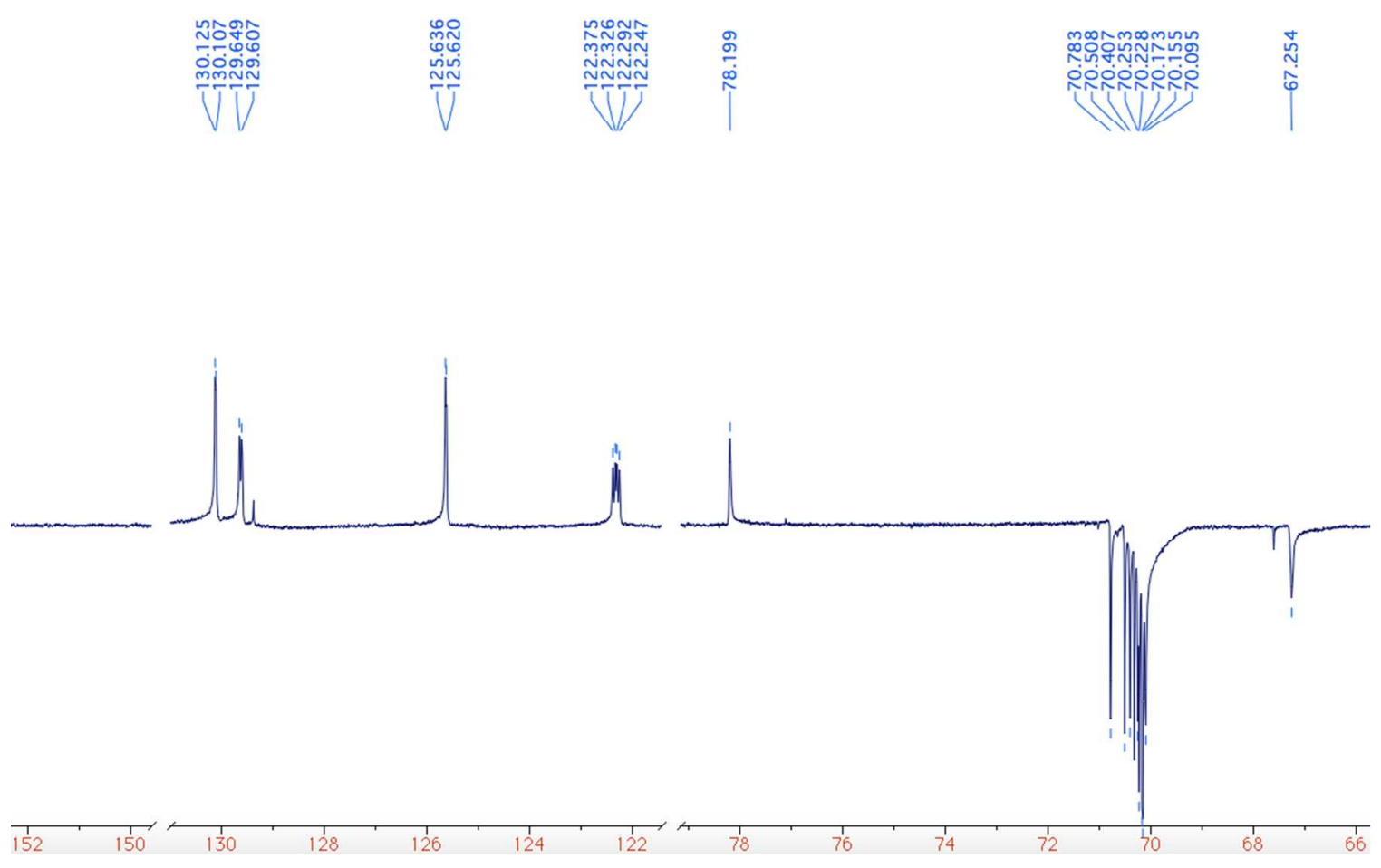

Figure S32. DEPT135 NMR spectrum ( $700 \mathrm{MHz}, \mathrm{CD}_{2} \mathrm{Cl}_{2}$ ) for complex 3. (Some of the spectrum has been removed for clarity). 


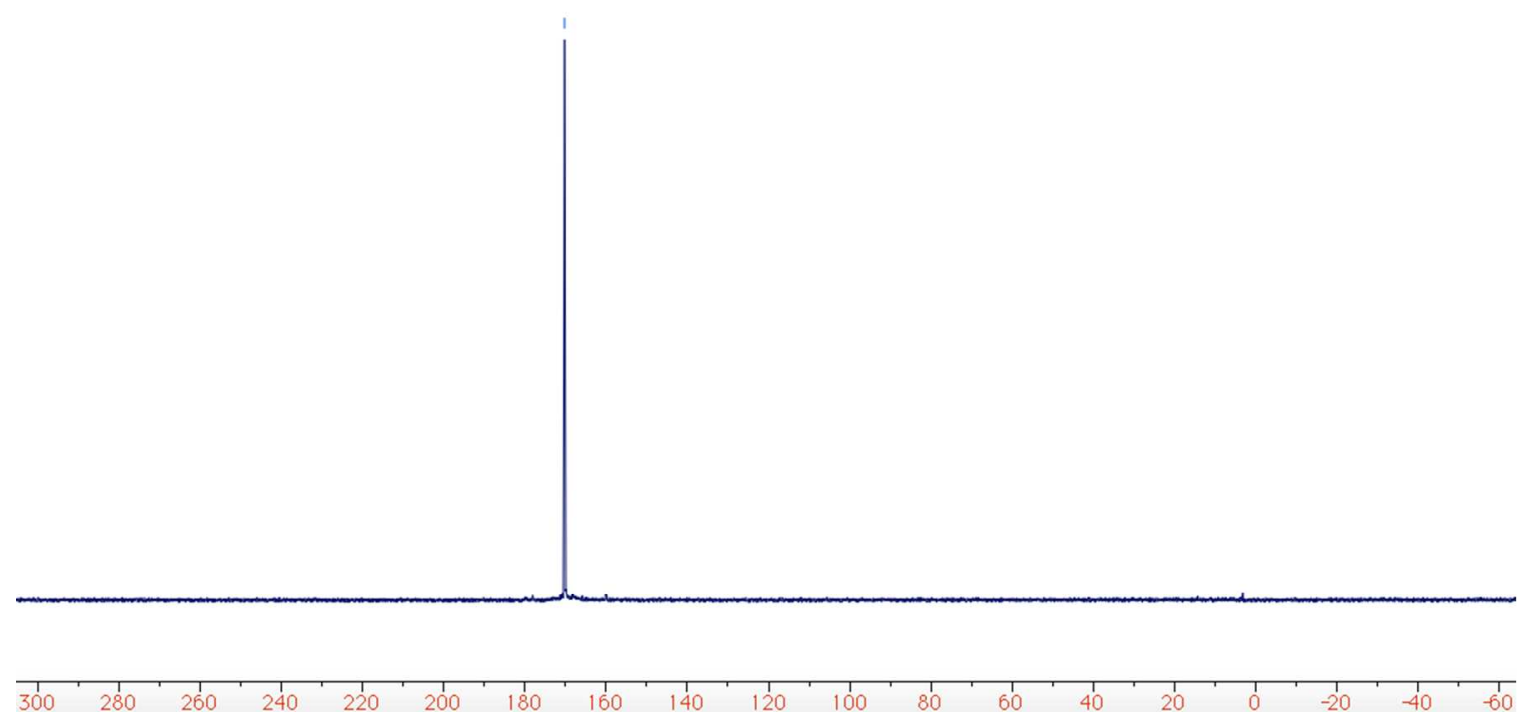

Figure S33. ${ }^{31} \mathrm{P}\left\{{ }^{1} \mathrm{H}\right\}$ NMR spectrum $\left(400 \mathrm{MHz}, \mathrm{CD}_{2} \mathrm{Cl}_{2}\right)$ for complex 3 . 


\section{(D) Additional NMR Spectra.}

\section{$1.2: 1$}

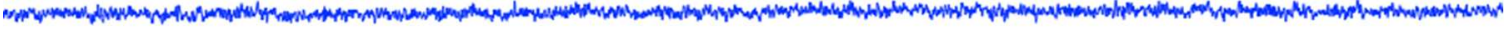

\section{$1.1: 1$}

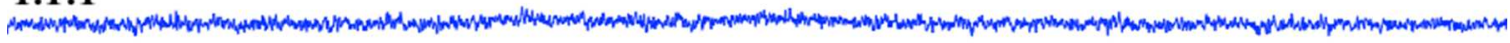

1:1

0.95:1

0.75:1

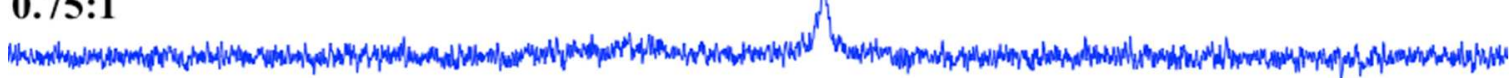

0.5:1

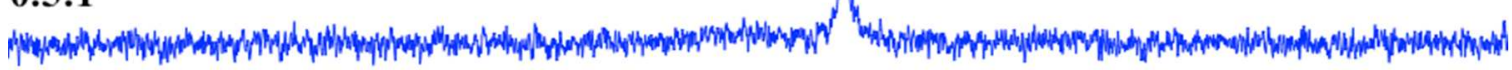
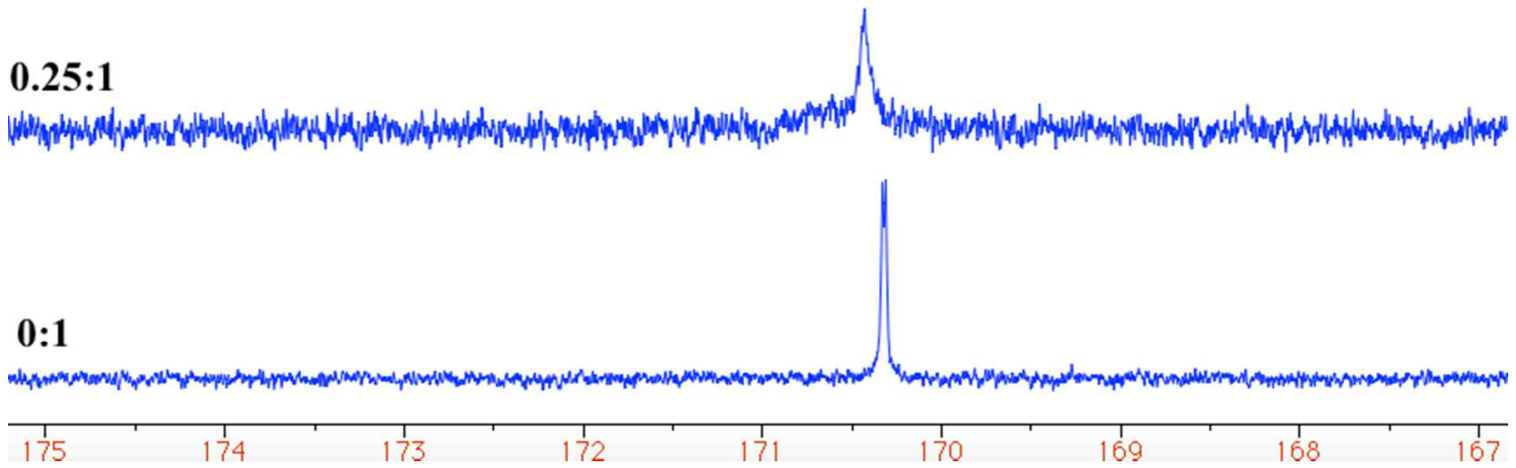

Figure S34. ${ }^{31} \mathrm{P}\left\{{ }^{1} \mathrm{H}\right\}$ NMR stack plot depicting observed changes in the ${ }^{31} \mathrm{P}\left\{{ }^{1} \mathrm{H}\right\} \mathrm{NMR}$ spectrum of 2 upon titration with $\mathrm{KB}\left(\mathrm{C}_{6} \mathrm{~F}_{5}\right)_{4}$. 


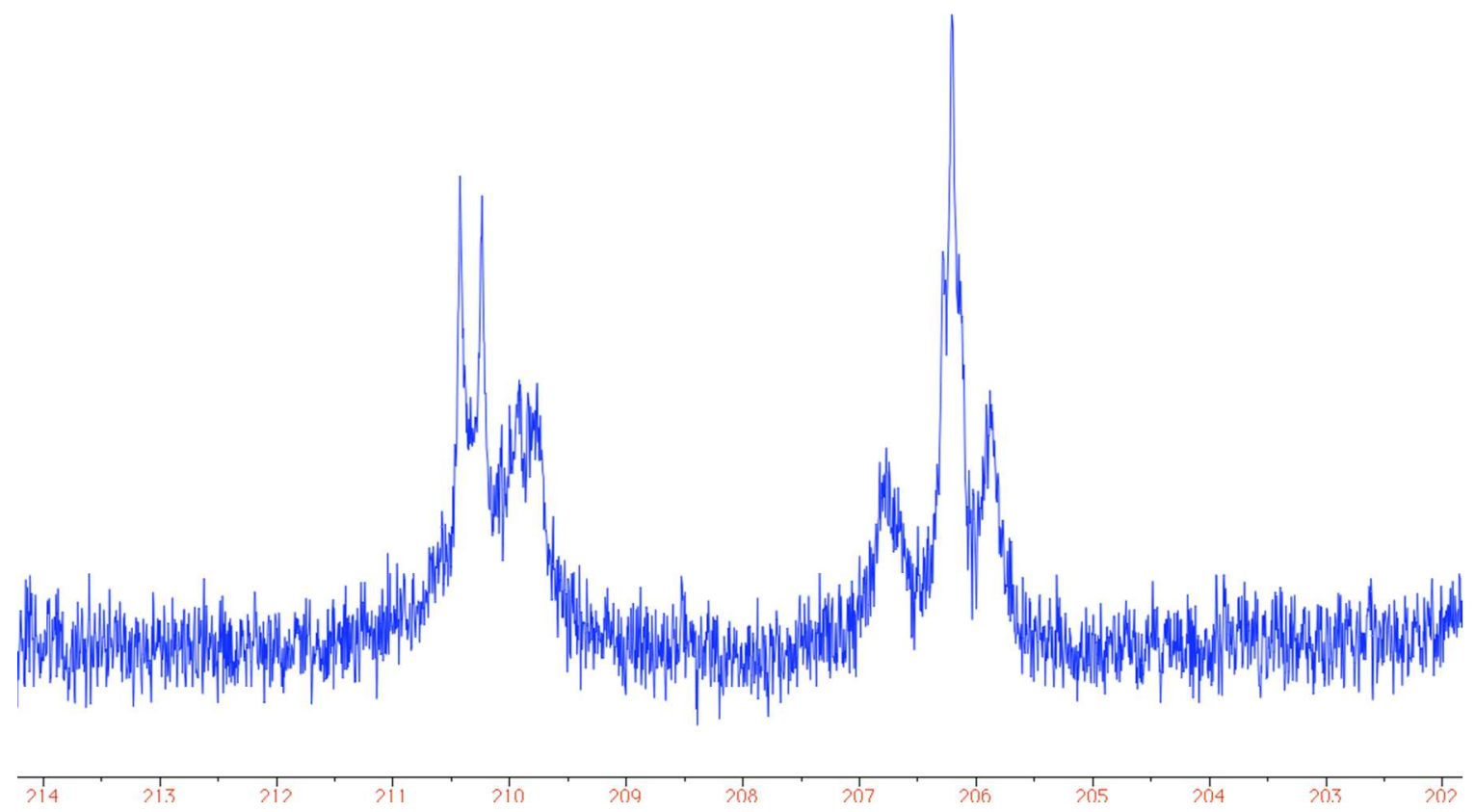

Figure S35. Carbonyl region of ${ }^{13} \mathrm{C}\left\{{ }^{1} \mathrm{H}\right\}$ NMR spectrum of $1: 13: \mathrm{K}^{+}$complex. Note similarities to the ${ }^{13} \mathrm{C}\left\{{ }^{1} \mathrm{H}\right\} \mathrm{NMR}$ spectrum of the $1: 12: \mathrm{Na}^{+}$complex (Figure 6). 

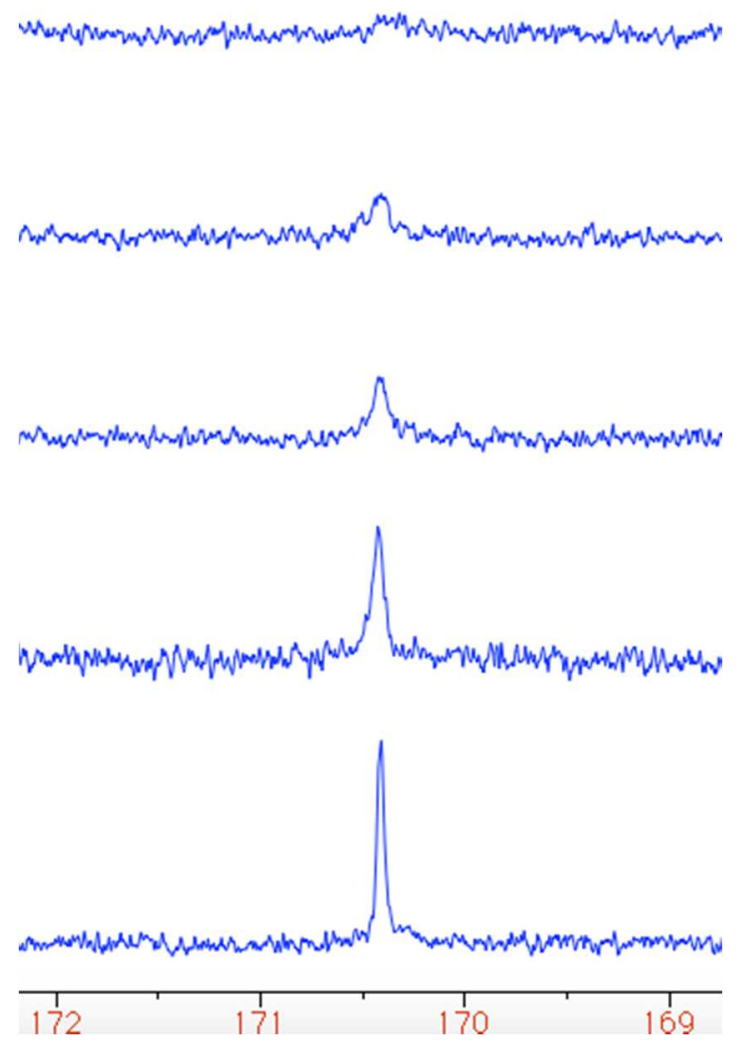

Figure S36. ${ }^{31} \mathrm{P}\left\{{ }^{1} \mathrm{H}\right\}$ NMR stack plot depicting observed changes in the ${ }^{31} \mathrm{P}\left\{{ }^{1} \mathrm{H}\right\} \mathrm{NMR}$ spectrum of 3 upon titration with $\mathrm{KB}\left(\mathrm{C}_{6} \mathrm{~F}_{5}\right)_{4}$.

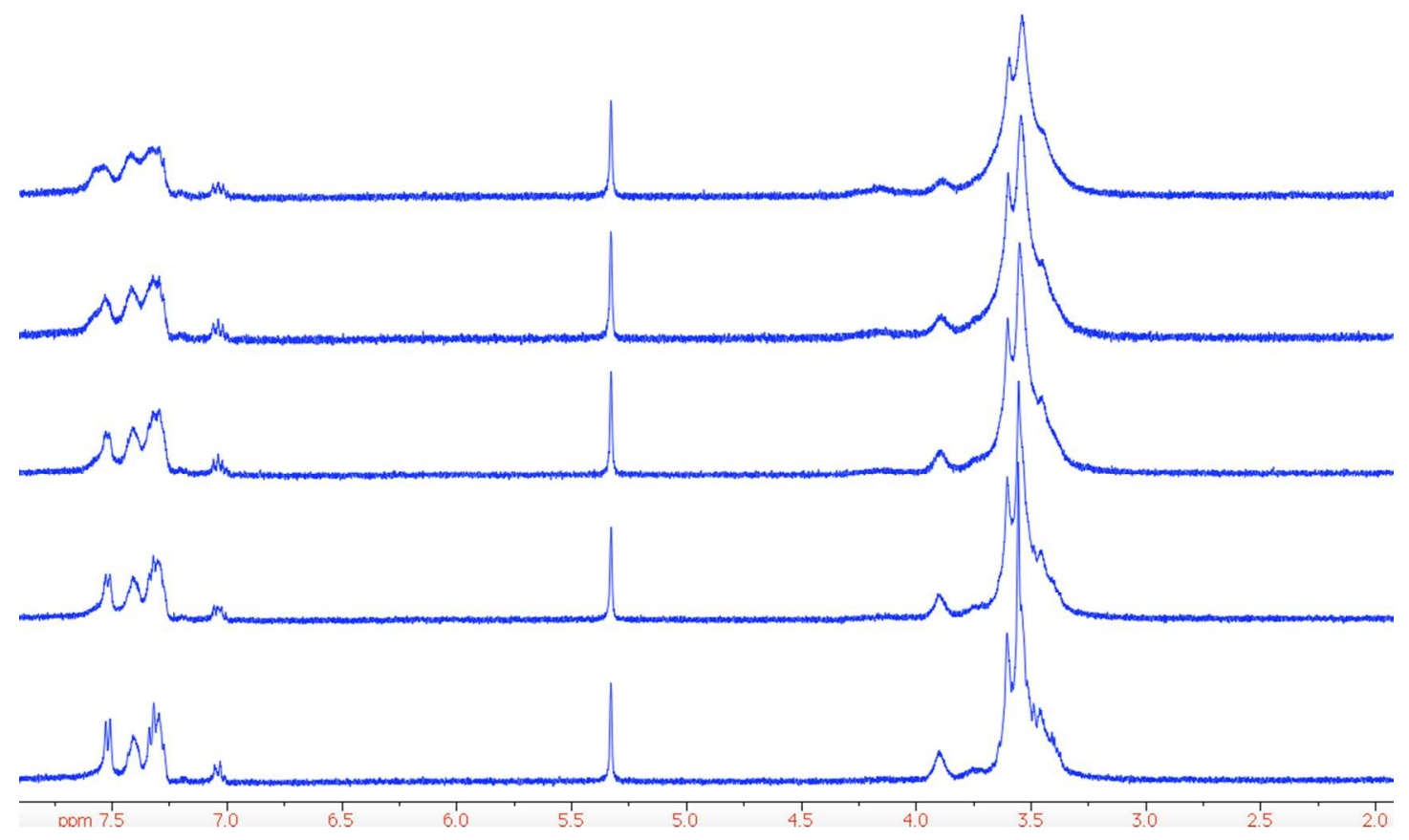

Figure S37. ${ }^{1} \mathrm{H}$ NMR stack plot depicting observed changes in the ${ }^{1} \mathrm{H}$ NMR spectrum of 3 upon titration with $\mathrm{KB}\left(\mathrm{C}_{6} \mathrm{~F}_{5}\right)_{4}$. 